\title{
Slipping fluids: a unified transient network model
}

\author{
Yogesh M. Joshi ${ }^{\mathrm{a}}$, Ashish K. Lele ${ }^{\mathrm{a}}$, R.A. Mashelkar, ${ }^{\mathrm{b}, *}$ \\ ${ }^{a}$ Chemical Engineering Division, National Chemical Laboratory, Pune 411008, India \\ ${ }^{\mathrm{b}}$ Council of Scientific and Industrial Research, Anusandhan Bhavan, 2 Rafi Marg, New Delhi 110001, India
}

Received 18 January 1999; received in revised form 26 April 1999

\begin{abstract}
Wall slip in polymer solutions and melts play an important role in fluid flow, heat transfer and mass transfer near solid boundaries. Several different physical mechanisms have been suggested for wall slip in entangled systems. We look at the wall slip phenomenon from the point of view of a transient network model, which is suitable for describing both, entangled solutions and melts. We propose a model, which brings about unification of different mechanisms for slip. We assume that the surface is of very high energy and the dynamics of chain entanglement and disentanglement at the wall is different from those in the bulk. We show that severe disentanglement in the annular wall region of one radius of gyration thickness can give rise to non-monotonic flow curve locally in that region. By proposing suitable functions for the chain dynamics so as to capture the right physics, we show that the model can predict all features of wall slip, such as flow enhancement, diameter-dependent flow curves, discontinuous increase in flow rate at a critical stress, hysteresis in flow curves, the possibility of pressure oscillations in extrusion and a second critical wall shear stress at which another jump in flow rate can occur. (C) 2000 Elsevier Science B.V. All rights reserved.
\end{abstract}

Keywords: Slip; Flow instabilities; Constitutive instability; Transient network model

\section{Introduction}

Wall slip in flowing polymeric solutions and melts has been investigated for the past several decades. Slip is seen in capillary flow (e.g. Refs. [1-3]), rectilinear flow (e.g. Refs. [4-6]), large amplitude oscillatory flow (e.g. Ref. [6]), and film flow (e.g. Ref. [7]). Slipping fluids exhibit many typical characteristics, such as drastic reduction of resistance to flow (e.g. Refs. [8,9]), the presence of a critical shear stress above which resistance to flow decreases (e.g. Ref. [3]), diameter-dependence of flow curves (e.g. Refs. [3,8,9]), surface distortions of the extrudate (melt fracture) (e.g. Refs. [2,10]) and the (apparent) violation of no-slip boundary condition close to the wall (e.g. Refs. [4,11]).

\footnotetext{
* Corresponding author. Tel.: +91-11-371-0472; fax: +91-11-371-0618.

E-mail address: dgcsir@csirhq.ren.nic.in (R.A. Mashelkar)
} 
Several different theoretical interpretations exist for wall slip. In the case of polymer solutions, apparent wall slip has been attributed to migration of macromolecules away from the wall under a stress gradient [8,12,13]. For melts, the mechanisms of constitutive (bulk) instability [14-16], desorption from the wall [17-19] and chain disentanglement at the wall [20] have been proposed for explaining wall slip. Theoretical formulations of each of these mechanisms differ considerably, thus making it difficult to ascribe the experimentally observed slip to any one of the mechanisms.

In this paper, we show that different mechanisms for slip can be unified in the framework of a transient network model. We specifically consider the case of capillary flow in which wall-polymer interaction is strong and show that the local stress-strain rate curve in the wall region can become nonmonotonic because of disentanglement of chains at the wall. The non-monotonic nature of the curve can appear again at higher stresses due to bulk disentanglement (constitutive instability). Our model successfully predicts the existence of a critical shear stress, hysteresis, flow enhancement, diameterdependent flow curves and the possibility of pressure fluctuations in controlled rate capillary experiments.

Wall slip in polymer solutions has been inferred from macroscopic measurements, such as flow enhancement and diameter-dependent flow curves. At a microscopic level, slip has been indirectly inferred by measuring the concentration profile near the wall or by directly measuring the velocity profile near the wall. Some important experimental studies have been summarized in Appendix A.

Several attempts have been made to propose stress-induced migration as the cause for apparent wall slip in polymer solutions (see Appendix B). However, the theories suffer from four main drawbacks. There is a fundamental difficulty in introducing thermodynamic arguments for stress induced migration in a flowing (non-equilibrium) system. Further, different theories predict contradictory trends for migration in capillary flows, as summarized in the first three rows of Appendix B. Also, even today there is no convincing experimental evidence for radial migration of polymer molecules in pipe flow. Finally, the predicted $L / D$ for slip to occur is still too high, when compared to experimental observations.

The flow anomalies due to wall slip observed in extrusion of polymer melts are in many ways similar to those in polymer solutions. For example, Vinogradov [21] reported flow-rate enhancement and diameter-dependent flow curves for extrusion of polybutadine. Pressure-drop oscillations and rough extrudate surface (melt fracture) are well known phenomena that occur in controlled flow extrusion. These phenomena have been extensively studied [22,3] and reviewed [23-26,58,82]. Appendix C summarizes the main experimental reports on wall slip in melts.

Adhesive failure at the wall has been proposed to be responsible for slip in polymer melt extrusion, since the energy of the wall-polymer interface is known to dramatically influence the slip behavior [1,22]. Polymer molecules adsorbed on the wall undergo sudden desorption above a critical stress and, hence, slip at the wall. Hill [19] has recently proposed a quasi-chemical model in which polymer chains near the wall undergo a dynamic adsorption-desorption process that is influenced by flow. The model predicts a critical wall shear stress at which large slip occurs by a sudden desorption of the chains from the wall.

Constitutive instability (of bulk material) has also been proposed as another mechanism for wall slip. This mechanism is related to a non-monotonic stress-strain rate relationship [14]. Doi-Edwards theory predicts that stress passes through a maximum and then decreases with a further increase in the shear rate, which leads to mechanical instability in steady shearing [27]. Modification of the Doi-Edwards 
theory [28] and other theories, such as Johnson-Segalman and Giesekus models [16] predict a local stress minimum following the maximum.

Recently, the wall slip problem in melts has been re-visited with renewed interest because of some interesting new insights developed by Brochard and de-Gennes [20]. They proposed that polymer chains adsorbed on the wall (in the mushroom region) undergo a coil to stretch transition at a critical shear stress. Since stretched molecules cannot entangle with the bulk molecules, the bulk slips past the stretched chains. They showed that the critical shear stress is given by,

$$
\tau_{\mathrm{w}}^{*}=\nu\left[\frac{k T}{\left(N_{\mathrm{e}}^{0.5} a\right)}\right]
$$

where $\nu$ is the number of chains per unit area grafted to the wall, $N_{\mathrm{e}}$ the entanglement distance and ' $a$ ' the Rouse length. Eq. (1) indicates that the critical wall shear stress $\left(\tau_{\mathrm{w}}^{*}\right)$ increases with temperature and grafting density (in the mushroom regime).

Migler et al. [4] and Leger et al. [26] experimentally observed slip in PDMS melts flowing on mica surfaces, on which chains were strongly adsorbed to create a low grafting density (mushroom) brush. Their experimental results on slip-length/slip velocity relations are in excellent agreement with predictions of Brochard and de-Gennes [20]. Wang and Drda [29] showed a discontinuous jump in the flow rate at a critical stress (similar to data of Vinogradov et al. [21]) for controlled pressure-drop extrusion of HDPE melt. They showed that the critical shear stress for HDPE extrusion increased with increase in temperature, which is in agreement with Brochard-de Gennes model [20]. Wang and Drda [3] argue that if sudden desorption was the governing mechanism for slip, then the critical stress should decrease with an increase in temperature.

Interestingly, Wang and Drda [3] also reported a second critical stress, at which the flow rate again increased discontinuously. The flow curves after this second critical stress do not show diameter dependence, unlike those after the first critical stress. The authors claimed that the second criticality might arise out of disentanglement within the bulk chains.

Kolnaar and Keller [10,30] have reported the existence of a narrow temperature range $\left(146-152^{\circ} \mathrm{C}\right)$ in which, above a certain piston speed, the extrusion pressure decreases significantly with a small increase in temperature. Beyond the temperature window, the pressure increased and showed oscillations accompanied by melt fracture. In situ wide angle X-ray diffraction results showed an anomalous hexagonal phase near the capillary wall (Van Bislen et al. [31]). The authors proposed that such a hexagonal phase is responsible for the slippage of polymer molecules at the wall, thereby causing a decrease in pressure. Till this day this remains the only direct experimental observation on chain stretching at the wall accompanying wall slip.

Returning to polymer solutions, it is interesting to point out that the recent experimental investigations on slip in concentrated solutions of high molecular weight polymers suggest that chain stretching and disentanglement at the wall seems to be responsible for the observed wall slip. Archer et al. [32] observed a large displacement of $1.5 \mu \mathrm{m}$ tracer particles at the stationary wall on the cessation of shear flow of high molecular weight entangled polystyrene solution. Riemers and Dealy [5,33] have observed slip above a critical shear stress in high molecular weight and narrow MWD polystyrene solution. Mhetar and Archer [34] have observed significant levels of slip during steady shearing (couette flow) of entangled polystyrene solutions. 
From the foregoing discussions it is evident that experimentally observed effects of wall slip (such as flow enhancement and diameter dependence of flow curves) are similar for both polymer solutions and melts. Different interpretations, such as migration, desorption, constitutive instability and chain extension exist to explain the wall slip. It is safe to assume that polymer migration may be ruled out for the case of concentrated polymer solutions, because of the problems listed earlier. It is also likely that different mechanisms are probably active in different regimes of experimental parameters. We believe that the chain stretching and desorption are the most plausible mechanisms for explaining wall slip in entangled solutions and melts.

In this work, it is our endeavor to develop the framework of a unified model to interpret the phenomenon of slipping fluids. We look at the capillary flow of a fluid, whose chains entangle to form a transient network. For such a fluid, polymer chains can adsorb on the wall through energetic interactions and the adsorbed chains can simultaneously entangle with the bulk chains. A transient network model can provide an appropriate basis to study the flow of such a fluid. We will show that the model can predict wall slip by chain disentanglement at the wall. Similarly, we will show in a later publication that the same model can also describe wall slip by the chain-desorption process.

An attempt to use a transient network model to describe the desorption process was indeed made earlier $[35,36]$. We will show that our approach is fundamentally different from the previous work. We will also show that a transient network model containing strain-dependent rates of formation and loss of junctions can predict all the typical characteristics of slip flow, namely flow enhancement, diameterdependent flow curves, critical wall shear stress and large disentanglement. We will also show quantitative comparisons between the experimental data and our model for the representative cases of polymer solutions and melts. Importantly, our model does not need an arbitrary slip velocity at the wall, nor the migration of polymer molecules from the wall. Further, we will provide insights into the dynamics of chain entanglement-disentanglement process in the bulk and at the wall and their relation with the existence of a critical shear stress.

\section{Theoretical}

We begin by outlining the framework of a unified model based on the transient network concept. Consider the physical picture near the wall as depicted in the schematic shown in Fig. 1. Polymer segments attached to the wall form a transient network with segments of the bulk chains. The segments can break away from the network by either disentanglement from the bulk chains or by desorbing from the wall. For simplicity of the analysis, it is assumed that a polymer molecule attaches to the wall at a single site only. If $P_{w}$ is the number of chains per unit area attached to the wall, $P$ the number of bulk polymer molecules per unit area coming in contact with the bare wall and $w$ the number of bare sites per unit area on the wall on which a molecule can be bonded, then the reaction of adsorptiondesorption can be written as

$$
P_{\mathrm{w}} \underset{k_{\mathrm{a}}}{\stackrel{k_{\mathrm{d}}}{\rightleftarrows}} P+w
$$

where, $k_{\mathrm{a}}$ and $k_{\mathrm{d}}$ are kinetic rate constants for adsorption and desorption reaction. From Eq. (2),

$$
\frac{\mathrm{d}\left[P_{\mathrm{w}}\right]}{\mathrm{d} t}=k_{\mathrm{a}}[P][w]-k_{\mathrm{d}}\left[P_{\mathrm{w}}\right]
$$




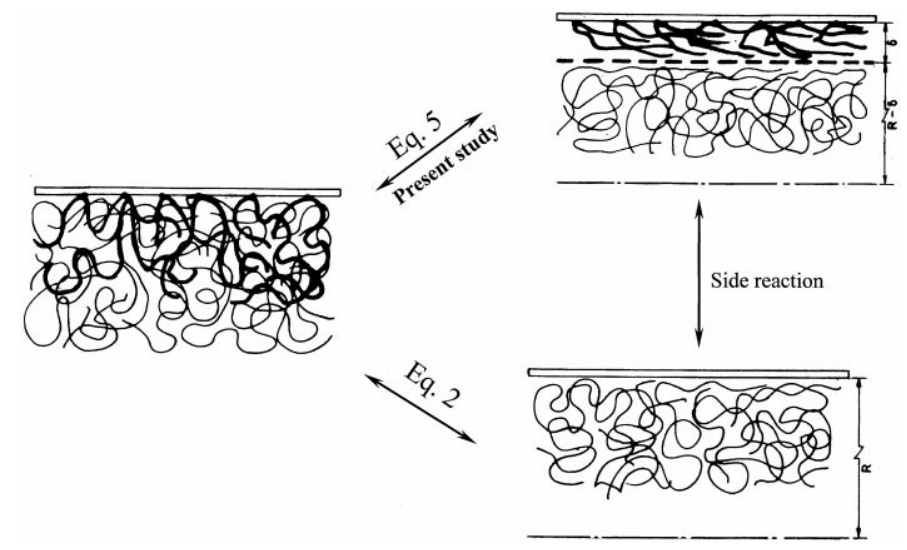

Fig. 1. Schematic representation of flow-induced disentanglement and debonding of polymer molecules attached to a wall. In case of disentanglement, the pipe is divided into two regions as shown in the figure.

Considering Eq. (3) to be at equilibrium, we get

$$
\phi=\frac{\left[P_{\mathrm{w}}\right]}{\left[w_{\mathrm{t}}\right]} \equiv \frac{k_{\mathrm{a}}[P]}{k_{\mathrm{a}}[P]+k_{\mathrm{d}}},
$$

where $\phi$ is the fraction of surface coverage, $w_{\mathrm{t}}$ the total number of sites per unit area to which polymer molecules can attach $\left(w_{\mathrm{t}}=P_{w}+w\right)$. $[P]$ can be assumed to be constant, because it is a very high value. Since the kinetics of adsorption and desorption are extremely fast as compared to the rheological time scale, it is appropriate to consider reaction (2) to be under equilibrium before the process of disentanglement starts.

The process of entanglement-disentanglement also can be written as a kinetic reaction as follows:

$$
P_{\mathrm{w}}^{\mathrm{e}} \underset{k_{2}}{\stackrel{k_{1}}{\rightleftarrows}} P_{\mathrm{w}}^{\mathrm{d}}
$$

where $P_{\mathrm{w}}^{\mathrm{e}}$ are the molecules which are attached to the surface and entangled with the bulk, while $P_{\mathrm{w}}^{\mathrm{d}}$ those attached to the wall but disentangled from the bulk. Also $P_{\mathrm{w}}=P_{\mathrm{w}}^{\mathrm{e}}+P_{\mathrm{w}}^{\mathrm{d}}$. Considering reaction (5) and using Eq. (4), the fractional surface coverage of the molecules that are entangled with the bulk can be written as

$$
\varphi=\frac{\left[P_{\mathrm{w}}^{\mathrm{e}}\right]}{\left[w_{\mathrm{t}}\right]}=\phi \frac{k_{2}}{\left(k_{1}+k_{2}\right)} .
$$

It can be seen from Eq. (6) that whichever be the governing mechanism for slip (i.e. desorption or disentanglement), the value of $\varphi$ decides the extent of total physical bonding between the wall and the bulk. It is assumed in this analysis that the adsorbed molecules do not detach from the wall $(\phi=1)$.

Consider the case of polymer molecules strongly adsorbed on the wall, say by hydrogen bonding. Flow induced desorption would require that the tension in the segment should exceed the adsorption force. The tension in the freely joined segment can be estimated as $F_{T} \sim k T / a \sqrt{N_{\mathrm{e}}} \sim k T / 10 a$ [20]. 
Here, $N_{\mathrm{e}}$ is the entanglement length. The force of adsorption can be estimated to be $F_{\mathrm{H}} \sim E_{\mathrm{H}} / a$, where the energy of hydrogen bonding $E_{\mathrm{H}} \sim \mathrm{O}(2 k T)$. Thus, $F_{\mathrm{T}} \ll F_{\mathrm{H}}$ and it is expected that chain stretching by flow would not significantly affect the adsorption-desorption dynamics. In this case, the network dynamics is expected to be governed by the entanglement-disentanglement process.

For the case of weak adsorption, for which $E_{\mathrm{H}} \ll \mathrm{O}(2 k T)$, the flow can significantly affect the adsorption-desorption kinetics, making it the governing mechanism for network dynamics. The above discussion also suggests that the 'side reaction' of desorption of disentangled chains can be neglected. This is because strongly adsorbed chains (e.g. PDMS on mica) have to be stretched much beyond the disentangled state to be desorbed by flow, and weakly adsorbed chains (e.g. on fluoropolymer-coated die) can be desorbed much before disentanglement. In this paper, we will only consider the case of strongly adsorbed chains. Hence, the following analysis considers the case of full surface coverage, that is $\phi=1$.

We will now combine this conceptual development with a transient network model. In transient network models, the contribution to stress is considered to be localized at entanglement points called junctions. A segment, which joins two junction points, is assumed to be a Gaussian spring. If the number of segments of type $i$ and length $Q$, that are created per unit time per unit volume at time $t$, is denoted by $L_{i}(Q, t)$ and the probability that the segments are destroyed is $\lambda_{i}^{-1}(Q, t)$, then the diffusion equation which determines the distribution function of such segments is given by [37]:

$$
\left.\frac{\partial \psi_{\mathrm{iN}}}{\partial t}=-\left(\frac{\partial}{\partial \underset{\sim}{\mathbf{Q}}} \cdot[\underset{\sim}{\underset{\sim}{\underset{\sim}{\alpha}}} \underset{\sim}{\mathbf{Q}}] \psi_{\mathrm{iN}}\right]\right)+L_{\mathrm{iN}}(Q, t)-\frac{\psi_{\mathrm{iN}}}{\lambda_{\mathrm{iN}}(Q, t)},
$$

where $\underset{\sim}{\mathbf{Q}}$ is the segment vector and $\underset{\approx}{\kappa}=(\underset{\sim}{\nabla v} \underset{\sim}{v})^{T}$ is the deformation gradient tensor. $\psi_{\mathrm{iN}}(\underset{\sim}{\mathbf{Q}}, t) \mathrm{d} \underset{\sim}{\mathbf{Q}}$ is number of segments per unit volume at time $\tilde{t}$ that have end-to-end vector in the range of $\mathrm{d} \underset{\sim}{\tilde{\mathbf{Q}}}$ at $\underset{\sim}{\mathbf{Q}}$. In the transient network model, the total stress is assumed to be the sum of contributions from individual segments The expression for the total stress is given by

$$
\underset{\approx}{\pi}=-\sum_{i} \underset{\sim}{H \underset{\sim}{\mathbf{Q}}\rangle_{i}}
$$

where $H$ is a spring constant.

The constitutive equations obtained from Eqs. (7) and (8) are given by

$$
\underset{\approx}{\tau_{i}}+\lambda_{i} \underset{\approx}{\stackrel{\nabla}{\tau_{i}}}=-k T \hat{L}_{i}^{\mathrm{eq}} \lambda_{i}^{\mathrm{eq}} \lambda_{i}(t) \underset{\approx}{\dot{\gamma}}-k T\left[\hat{L}_{i}(t) \lambda_{i}(t)-\hat{L}_{i}^{\mathrm{eq}} \lambda_{i}^{\mathrm{eq}}\right] \underset{\approx}{\delta}
$$

and

$$
\underset{\tau}{\tau}=\sum_{i} \underset{\approx}{\tau_{i}}
$$

Here, $\hat{L}_{i}^{\mathrm{eq}}$ and $\lambda_{i}^{\mathrm{eq}}$ are the equilibrium creation and loss terms and $\underset{\sim}{\stackrel{\nabla}{\tau}}$ is the upper convected derivative. The modulus $G_{0 i}$ is defined as

$$
G_{0 i}=k T \hat{L}_{i}^{\mathrm{eq}} \lambda_{i}^{\mathrm{eq}}
$$


Next, the creation and loss functions are defined as

$$
\begin{aligned}
& \hat{L}_{i}(t)=f_{i}(t) \hat{L}_{i}^{\mathrm{eq}}(t), \\
& \lambda_{i}(t)=\lambda_{i}^{\mathrm{eq}} / g_{i}(t) .
\end{aligned}
$$

We further assume for the sake of simplicity that the network segments are of only one type $(i=1)$ and, henceforth, drop the subscript $i$. Inserting Eqs. (11) and (12) into Eq. (9) and non-dimensionalizing with respect to the following parameters for pipe flow,

$$
\underset{\approx}{\tau^{*}}=\frac{\underset{\tau}{\tau}}{G_{0}}, t^{*}=\frac{v_{\mathrm{m}}}{R} t, r^{*}=\frac{r}{R}, \underset{\approx}{\dot{\gamma}^{*}}=\lambda \underset{\approx}{\lambda \dot{\gamma}}, z^{*}=\frac{z}{R}, \underset{\sim}{\nabla^{*}}=R \underset{\sim}{\nabla}, \underset{\approx}{\tau^{*}}=\frac{G_{0} v_{\mathrm{m}}}{R} \underset{\approx}{\sim}
$$

we get,

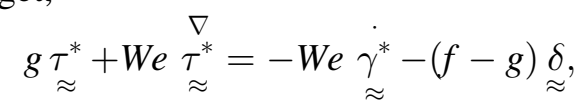

where, superscript $*$ indicates non-dimensionalized variables, $\lambda$ the relaxation time, $R$ the radius of the pipe, $v_{\mathrm{m}}$ the maximum velocity, $W e=\lambda v_{\mathrm{m}} / R$ the Weissenberg number.

We now consider the fully developed pipe flow problem for which, $v=v\left(v_{\mathrm{z}}\right), v_{\mathrm{z}}=v_{\mathrm{z}}(r), \mathrm{P}=\mathrm{P}(z, r)$, $\underset{\approx}{\tau}=\underset{\approx}{\tau}(r)$. The equation of motion simply reduces to

$$
\begin{aligned}
& 0=-\frac{\partial \mathrm{P}}{\partial r}-\frac{1}{r} \frac{\partial\left(r \tau_{r r}\right)}{\partial r}+\frac{\tau_{\theta \theta}}{r}, \\
& 0=-\frac{\partial \mathrm{P}}{\partial z}-\frac{1}{r} \frac{\partial\left(r \tau_{r z}\right)}{\partial r} .
\end{aligned}
$$

It can be easily shown that the transient network model predicts $\tau_{r r}=\tau_{\theta \theta}$ (refer Eqs. (19) and (20)). Thus, from Eqs. (15) and (16)

$$
\mathrm{P}(r, z)=F(r)+G(z),
$$

and, hence, from Eq. (16):

$$
\tau_{r z}=-\frac{r}{2} \frac{\partial \mathrm{P}}{\partial z}
$$

For pipe flow, the constitutive equation (Eq. (14)) can be written in component form as:

$$
\begin{aligned}
& g \tau_{r r}^{*}=-f+g, \\
& g \tau_{\theta \theta}^{*}=-f+g, \\
& g \tau_{z z}^{*}-2 W e \tau_{r z}^{*} \frac{\partial v_{z}^{*}}{\partial r^{*}}=-f+g, \\
& g \tau_{z z}-W e \tau_{r r}^{*} \frac{\partial v_{z}^{*}}{\partial r^{*}}=-W e \frac{\partial v_{z}^{*}}{\partial r^{*}} .
\end{aligned}
$$

Eqs. (18)-(22) represent the final set of equations for the pipe flow problem. 
The solution of this set of equations requires description of functions $f$ and $g$. Since the kinetics of entanglement-disentanglement are extremely complex, a theoretical derivation of the functional form of $f$ and $g$ has not been possible so far. Several empirical forms have been suggested [38-41]. In principle, it is possible to formulate the creation- and loss-rate functions as a function of the segment length $\mathbf{Q}$. However, any functional dependence of this nature gives rise to complex coupled constitutive equations, which require demanding computations. In a simplified approach proposed by Ahn-Osaki $[41,42]$, the complexities are overcome by assuming that the creation and loss rates are functions of the effective strain

$$
\begin{gathered}
f=\exp \left(a \gamma_{e}\right), \\
g=\exp \left(b \gamma_{e}\right),
\end{gathered}
$$

where

$$
\gamma_{\mathrm{e}}=\frac{\tau_{11}-\tau_{22}}{2 \tau_{12}}
$$

Deeper mechanistic considerations of the problem at hand suggest the following:

- Because of adsorption of chains on the wall and the possibility of them getting stretched more easily as compared to chains in the bulk, the dynamics of entanglement and disentanglement near the wall are different from those in the bulk.

- Consequently, the capillary can be divided into two domains, namely, the bulk and the wall. The wall domain can be assumed to be an annulus of diameter equal to that of the pipe and thickness of the order of the radius of gyration of the molecule (see Fig. 1). The wall domain is significant till the molecules are attached to the wall.

- On stretching, the rates of creation and loss of entanglements also increase to a point of nearly complete disentanglement, after which the rates remain constant.

- Desorption of the molecules from the wall does not happen even at nearly complete disentanglement. As discussed earlier, this will hold for strongly adsorbed molecules.

Any of the mathematical forms of $f$ and $g$ will make the transient network model phenomenological to a certain extent. The foregoing arguments suggest that the formation and loss rates should have an ' $S$ ' shaped functional nature with respect to the effective strain. We propose a new empirical function for creation and loss rates,

$$
\begin{aligned}
& f=\frac{F_{f}}{2}\left(1+\operatorname{erf}\left(\frac{\gamma_{\mathrm{e}}-\alpha_{f}}{\theta_{f}}\right)\right), \\
& g=\frac{F_{g}}{2}\left(1+\operatorname{erf}\left(\frac{\gamma_{\mathrm{e}}-\alpha_{g}}{\theta_{g}}\right)\right),
\end{aligned}
$$

where erf $(x)=2 / \sqrt{\pi} \int_{0}^{x} \exp \left(-\xi^{2}\right) \mathrm{d} \xi$. The model parameters are $F_{f}, F_{g}, \theta_{f}, \theta_{g}$. The parameters $\alpha_{f}, \alpha_{g}$ are fitted such that $f=g \equiv 1$, at $\gamma_{\mathrm{e}}=0$.

The main difference between the above functions and the Ahn-Osaki exponential functions is that the creation and loss rates become asymptotically constant at high strains, at which the molecules might be sufficiently stretched. This behavior predicts a plateau at infinite shear rate, which is not possible 
with the Ahn-Osaki exponential function. As mentioned earlier, the creation- and loss-rate functions proposed here are purely empirical in nature. We proceed now to solve the capillary flow problem by using these functional forms and show that wall slip can be successfully predicted.

\section{Results and discussion}

\subsection{Important general predictions}

The five simultaneous equations (Eqs. (18)-(22)), i.e. the equation of motion and the constitutive equation can be elegantly simplified to give one main equation in terms of the effective strain as follows:

$$
G_{0} \gamma_{\mathrm{e}} \frac{f}{g}=-\frac{r}{2} \frac{\partial P}{\partial z} \equiv \tau_{r z}=r^{*} \tau_{w},
$$

where $f$ and $g$ are explicit function of $\gamma_{\mathrm{e}}$ as discussed in the previous section. This is the main equation of the paper. It describes the relation between the shear stress and the strain on a flowing fluid element. It can be shown that this equation holds not only for capillary flow but can be derived for other simple shear flows, such as in cone and plate or couette geometries (see Appendix D).

We consider here the case of steady state capillary flow under a controlled pressure gradient. As discussed in Section 2, the capillary is divided into two domains, namely the annular wall region ( $r=R-\delta$ to $r=R$ ) of thickness $\delta=10^{-8} \mathrm{~m}$ to account for attached chains and the remaining bulk region ( $r=0$ to $r=R-\delta$ ). For a numerical solution of Eq. (28), the bulk region is divided into 100 nodes and the wall annular region divided into four nodes. Eq. (28) is solved for obtaining the strain, $\gamma_{\mathrm{e}}$, at each radial position using the bisection method. In some calculations, the capillary is not divided into two domains, but its cross section is directly divided into 100 nodes from $r=0$ to $r=R$. The velocity at each radial position, $r$, is calculated from the effective strain by the following equation using a simple finite difference scheme as follows:

$$
\frac{\partial v_{z}^{*}}{\partial r}=\frac{g \gamma_{\mathrm{e}}}{W e R}
$$

Fig. 2 shows prediction of Eq. (28) for a typical set of model parameters. The stress-strain curves for the wall region can be seen along with creation and loss functions plotted with strain. The stress-strain curve for the wall region shows a non-monotonic behavior as the effective strain increases. The stress increases, then goes through a maximum, followed by a minimum, after which it increases continuously. A plot of stress-shear rate also follows the same pattern, but is shifted on the abscissa.

The origin of the non-monotonic stress-strain curve lies in the comparative rates of entanglement and disentanglement of chains at the wall. Fig. 2 also shows the functions $f$ and $g$ which are described by Eqs. (26) and (27). For low effective strain (low shear rates), the $f$ and $g$ values are small and close to their equilibrium value of unity. Thus, in the limit of zero strain, the stress increases linearly with strain. With increasing effective strain (shear rates), the strongly adsorbed chains at the wall stretch more easily than those in the flowing bulk do. The rate of disentanglement increases rapidly once a certain 


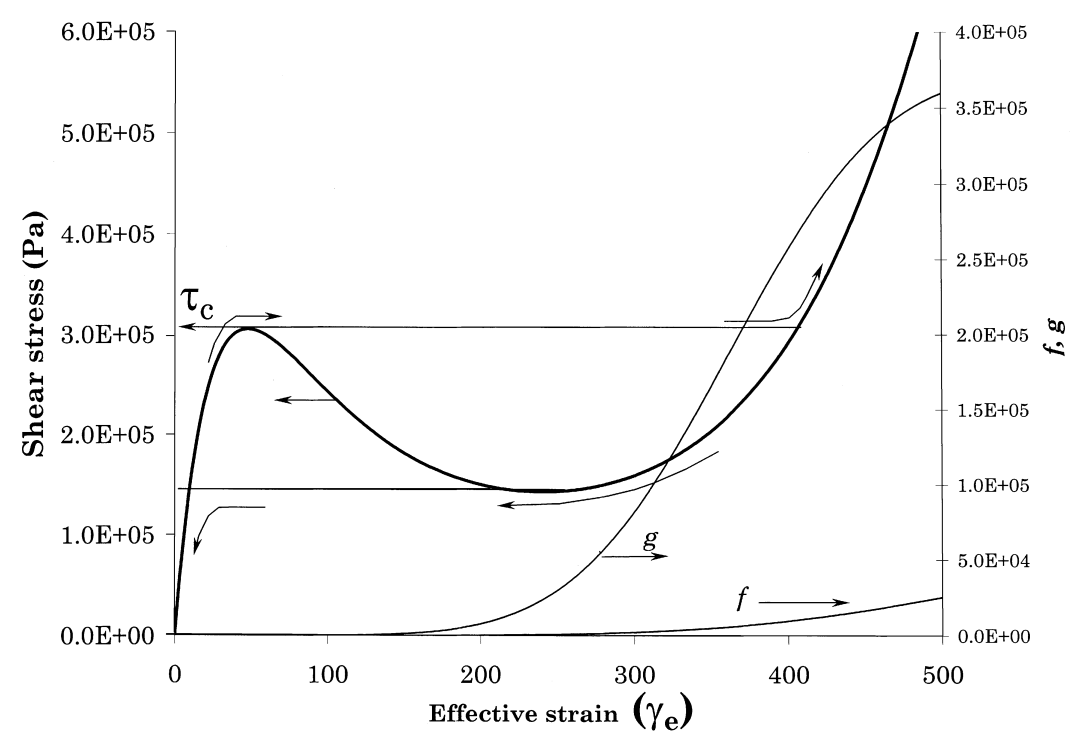

Fig. 2. Predictions of Eq. (28) in annular (wall) region. Non-monotonic curve in the wall region shows hysteresis. Also, behavior of $f$ and $g$ functions proposed in this paper (Eqs. (26) and (27)) are plotted on the right-hand side. The $f$ and $g$ functions are used to plot the stress-strain curve in the wall region.

effective strain is reached until the chains almost completely disentangle. Simultaneously, the rate of entanglement also increases in the stretched chains, as more sites are available for possible junction formation. The rates of entanglement and disentanglement balance each other at a state in which the number of entanglement points is very small and the chains are stretched. This is similar to the 'marginal state' proposed by Brochard and de Gennes [20]. The rapid rise in disentanglement rate causes the stress to decrease first, which gives rise to the maximum in stress. As the entanglement rate increases, the stress increases again; thus giving rise to the minimum in stress. At higher strains, $f$ and $g$ remain constant and the stress again increases linearly with strain.

The effect of disentanglement of adsorbed chains can be seen directly by recognizing that the lefthand side of Eq. (28) can be written as $G_{0} \gamma_{\mathrm{e}} f / g=G_{0} \gamma_{\mathrm{e}} n$, where $n$ is the normalized number of steady state network junctions. Thus, the $Y$-axis of Fig. 2 can be written as $G_{0} \gamma_{\mathrm{e}} n$. It is now clear that, as the stress decreases after the maximum, it is $n$ which decreases (i.e. disentanglement of chains). At larger strain, when $n$ remains constant the increase in stress corresponds to an increase in $\gamma_{\mathrm{e}}$.

Eq. (28) can also be written as

$$
\tau_{r z}=\frac{G_{0} \gamma_{\mathrm{e}} f}{g} \equiv\left(\phi n_{0} k T\right) n \gamma_{\mathrm{e}}
$$

where $n_{0}$ is the equilibrium number of entanglements of the adsorbed chains with the bulk chains per unit volume under no-flow condition, and $n_{0} n$ the total number of entanglements under full surface coverage $(\phi=1)$. Since the adsorption-desorption time scale is much smaller compared to the rheological time scale, $\phi n_{0}$ denotes the 'equilibrium' number of entanglements under flow conditions. In this study, we have considered the surface coverage $\phi$ to remain constant $(\phi=1)$. Eq. (30) shows 
that the wall shear stress is directly proportional to the temperature and to the surface coverage $\phi$. Both of these predictions are in qualitative agreement with the prediction of Brochard and de Gennes [20] [see Eq. (1)].

Non-monotonic stress/shear rate curves have been predicted by other models, as discussed in Section 1. For example, the Doi-Edwards model predicts a maximum in stress. A modification of this model by Mcleish and Ball [28] predicts both, a stress maximum and a stress minimum. They found that, above a critical shear stress, there would be a radial discontinuity in the flow rate and they assumed that the stable interface would exist at the minimum possible radius from the center. Similarly, the Johnson-Segalman model also predicts a stress maximum and minimum [16]. However, a fundamental difference between these predictions and our work is that the non-monotonic nature of the stress-strain (or strain rate) curves shown in Fig. 2 is due to disentanglement of chains at the wall and not in the bulk. In fact, our model also predicts a similar curve for bulk chains at much higher stresses, the implications of which will be discussed later.

It is pertinent to state that non-monotonic curves have also been observed experimentally. Akay [43] has observed non-monotonic flow curve for reinforced PP through a series of capillaries. Kissi and Piau [2] observed a non-monotonic pressure/flow rate relationship for PDMS melts, and Kolnaar and Keller [44] have reported a non-monotonic curve for uncorrected pressure vs. apparent wall shear rate.

Fig. 2 can be used to predict the flow curve, i.e. wall shear stress $\left(\tau_{\mathrm{w}}\right)$ vs. apparent shear rate $\left(\dot{\gamma}_{\mathrm{a}}=4 Q / \pi R^{3}\right)$, where the flow rate can be obtained by integrating Eq. (29). Thus, an increase in strain, $\gamma_{\mathrm{e}}$, is analogous to an increase in flow rate, $Q$. The region of the non-monotonic stress-strain curve in Fig. 2 in which the stress decreases with strain is a domain of unstable flow. Thus, if capillary flow experiments are carried out under controlled flow-rate conditions, i.e. along the abscissa of Fig. 2, then it is possible to travel through the unstable region. In such a case, the model would, in principle, predict pressure oscillations. However, if the experiments are carried out under controlled pressure-drop conditions, then the model predicts the existence of a critical wall shear stress at which a sudden jump in flow rate will be observed. Moreover, a hysteresis effect is also predicted. With increasing shear stress a 'top-jump' is possible, while with decreasing shear stress the system would probably show a 'bottom-jump' as indicated in Fig. 2.

It is important to note here that a multi-valued curve, such as that shown in Fig. 2, is not a necessary condition for the prediction of certain slip-characteristics, such as flow enhancement and diameter dependence. A difference in the dynamics of entanglement and disentanglement between bulk chains and wall chains is sufficient to predict flow enhancement and diameter-dependent flow curves.

Finally, it is interesting to note that the stress-strain diagram of Fig. 2 is qualitatively very similar to those observed in mechanical testing of solid polymers. In the limit of zero strain, the stress is linearly proportional to strain similar to Fig. 2. The 'yield' point in Fig. 2 occurs when the wall chains disentangle and stretch. At higher strains any further stretching of the disentangled chains requires increasing force, which is similar to the 'strain-hardening' phenomenon.

\subsection{Polymer solutions}

We now begin quantitative comparisons between model and experiments by analyzing experimental data on polymer solutions. As an example, we consider the data of Cohen and Metzner [8] for $0.5 \%$ aqueous hydrolyzed polyacrylamide (PAm) solution. The molecular weight of PAm is in the range of 


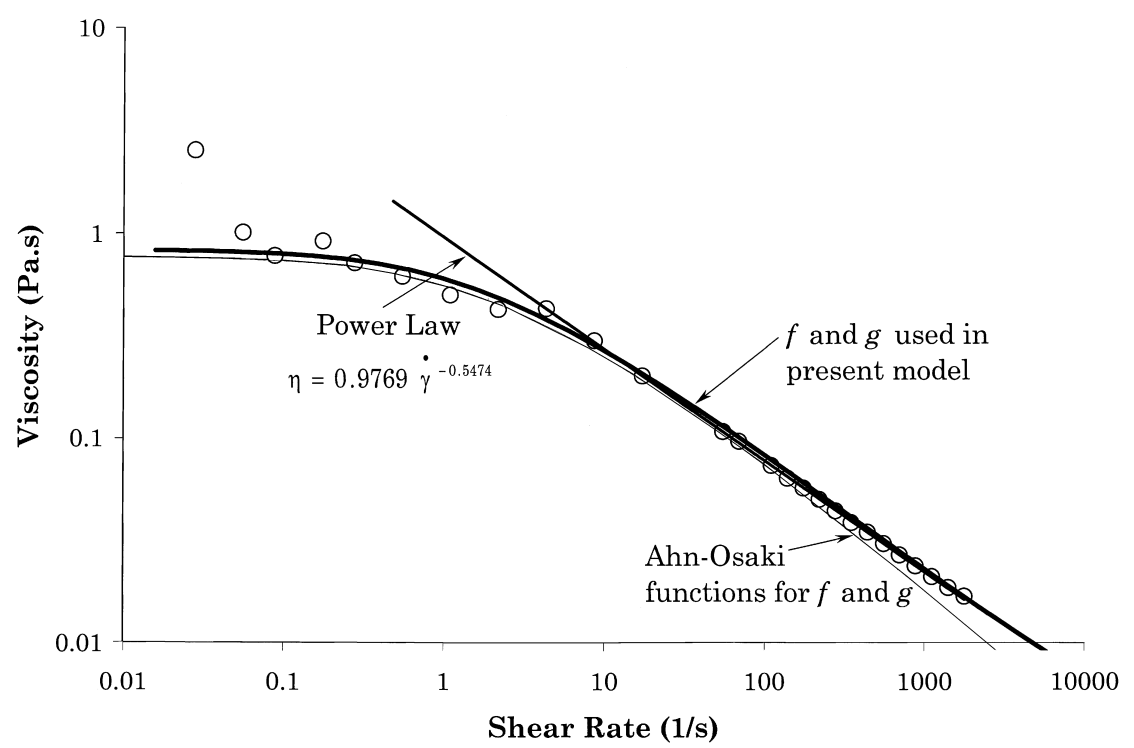

Fig. 3. Fit of the different models to viscosity-shear rate data of Cohen [45]. (1) Model parameters for transient network model using the Ahn-Osaki functions are $G_{0}=0.128, \lambda=60, a=0.12215$ and $b=0.1$; (2) Model parameters for transient network model using Eqs. (26) and (27) are $G_{0}=0.3, \lambda=2.25, F_{f}=50000, F_{g}=3000, \theta_{f}=20$, and $\theta_{g}=20$; and (3) for the power-law model, $n=0.453$ and $m=0.977$.

0.8-4.5 million [45]. The critical concentration $C^{*}$ can be calculated following Kulicke et al. [46]

$$
C^{*}=\frac{1.8 \times 10^{-25} \mathrm{M}}{\left(\left\langle R_{g}^{2}\right\rangle^{3 / 2}\right)} .
$$

Substituting the values of the radius of gyration as $\sqrt{\left\langle R_{g}^{2}\right\rangle}=10^{-10} \mathrm{M}^{1 / 2} / \sqrt{6}$ in meters [47], $C^{*}$ is found to be between $0.13 \%$ and $0.3 \%$, which indicates that $C>C^{*}$ and, hence, network theories can be used for data analysis.

We will first consider the $f$ and $g$ functions proposed by Ahn and Osaki [Eqs. (23) and (24)] to solve Eq. (28). The Ahn-Osaki model consists of four parameters namely, $a, b$, relaxation time $\lambda$, and modulus $G_{0}$. In order to obtain realistic values of these parameters, we have fitted the Ahn-Osaki model to viscosity/shear rate data of Cohen [45] for the PAm solution. Fig. 3 shows the fit of a power law model $(n \sim 0.453)$ and that of the transient network model with the Ahn-Osaki $f$ and $g$ functions.

Fig. 4 shows the predicted non-dimensional velocity profile for pipe flow using the AhnOsaki transient network model, the power law model and a Newtonian model at different pressure drops. As expected, the velocity profile predicted by the Newtonian and the power law models is pressure-drop independent, whereas that predicted by the network model is pressure drop dependent. It can be seen from Fig. 4 that the velocity gradient at the wall for the network model increases with pressure drop.

Using the same model parameters, the volumetric flow rate $Q$ is plotted against wall shear stress $\tau_{\mathrm{w}}$ in Fig. 5. The prediction of the transient network model with the Ahn-Osaki's $f$ and $g$ functions lie 


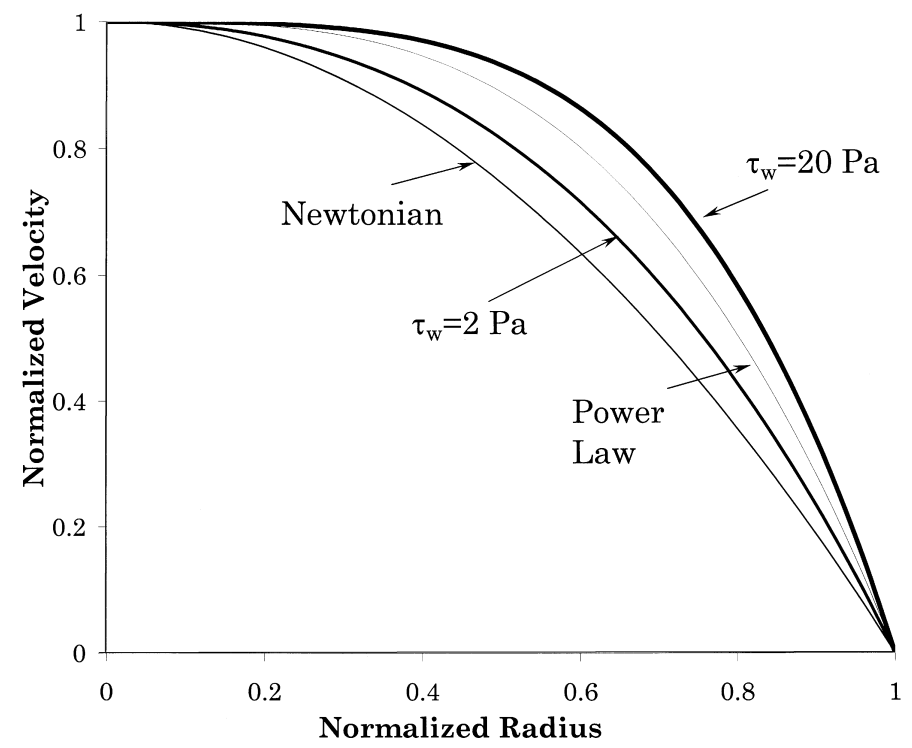

Fig. 4. Velocity profiles predicted using model parameters for the Ahn-Osaki functions as given in Fig. 3. Power-law model profile used in Fig. 3 and Newtonian profile are also shown.

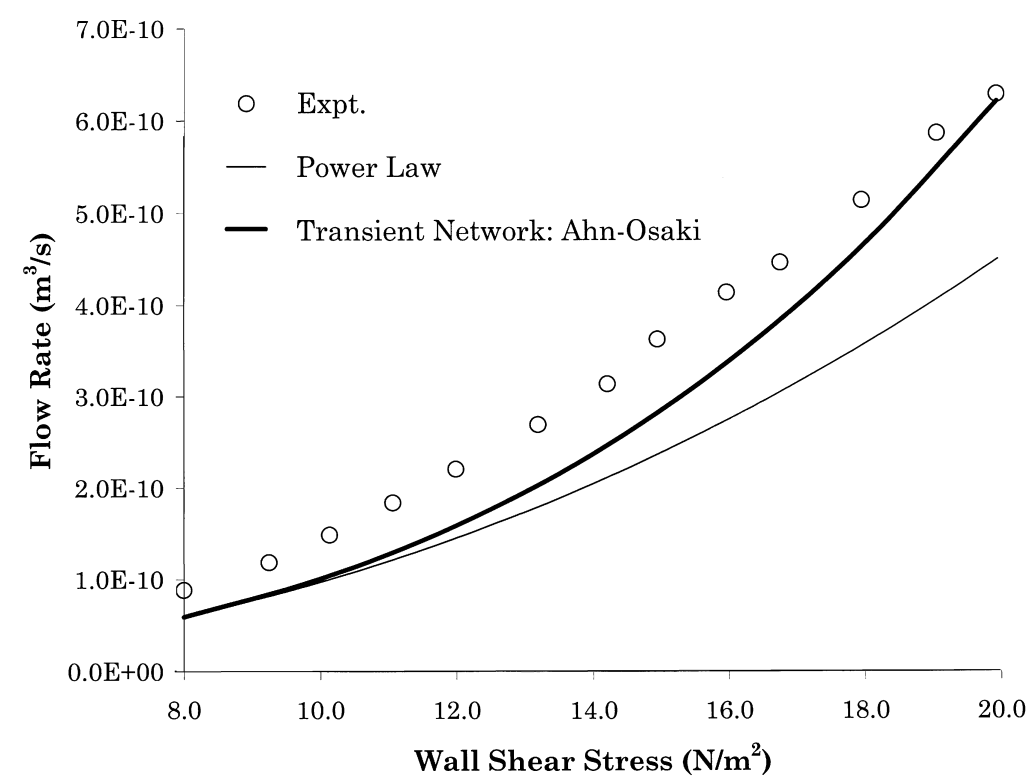

Fig. 5. Prediction of flow rate vs. wall shear stress for the power-law model and the transient network model using the AhnOsaki functions. Model parameters are the same as given in Fig. 3. Points represent experimental data [8].

above the power law prediction, and are in good agreement with the experimental data. Thus, the network model shows an apparent 'flow enhancement' at the same pressure drop over the power law model. This might be interpreted as slip-like behavior. 
Although the above analysis predicts a pressure-drop dependence of the velocity profile, it fails to predict a radius dependence. Since effective strain $\left(\gamma_{\mathrm{e}}\right)$ is a function of non-dimensionalized radius (see Eq. (28)), Eq. (29) can be integrated to give

$$
Q=\frac{2 \pi R^{3}}{\lambda} \int_{0}^{1} r^{*}\left(\int_{0}^{r^{*}} g \gamma_{\mathrm{e}} \mathrm{d} r^{*}\right) \mathrm{d} r^{*}
$$

It is clear that the apparent shear rate $\left(\dot{\gamma}_{\mathrm{a}}=4 Q / \pi R^{3}\right)$ is independent of $R$.

It can be concluded from the above analysis that, if the dynamics of entanglement and disentanglement are the same in the bulk and near the wall, results of the transient network model fail to show a radius dependence of the flow curves. Also, though the model predicts an increasing velocity gradient with increasing pressure drop, it still does not show a 'slip' velocity very close to the wall as observed experimentally [11].

The above discussion suggests that the behavior of the network at the wall might be different from that in the bulk. We, therefore, conceive the capillary as being divided into two domains, namely, an annular wall region having a thickness of the order of radius of gyration of the polymer molecule $\left(\sim 10^{-8} \mathrm{~m}\right)$, and the remaining space (bulk). It should be noted here that significance of the annular region is only to take into account different dynamics of attached molecules from that of the bulk. As discussed in Section 2, we assume that the dynamics of entanglement and disentanglement are given by Eqs. (26) and (27) and that the model parameters $F_{f}, F_{g}, \theta_{f}, \theta_{g}$, the relaxation time $\lambda$ and the modulus $G_{0}$ have different values in the bulk and in the wall region. Eqs. (28) and (29) are solved in the two regions with different model parameters, such that continuity in velocity and shear stress is maintained at the boundary of the two domains. Fig. 3 shows the fit of the transient network model using creation and loss functions given by Eqs. (26) and (27) to the viscosity/shear rate data of Cohen [45] for the PAm solution. We use the parameters obtained by this fit for the bulk domain during capillary flow. The reason for this is that the stress levels in cone-plate viscometric data are well below the critical stress, so that the dynamics of chains in the bulk and in the wall regions are the same. Therefore, although, in principle, the bulk and wall regions can exist for a cone-plate geometry, they are indistinguishable under the given experimental conditions.

Fig. 6 shows model calculations of apparent shear rate $\left(\dot{\gamma}_{\mathrm{a}}=4 Q / \pi R^{3}\right)$ vs. wall shear stress $\left(\tau_{\mathrm{w}}\right)$ compared with the experimental data of PAm [45]. The parameters for the bulk domain are obtained as discussed earlier. The parameters in the annular wall domain are obtained by fitting to the experimental points for $D=0.109 \mathrm{~cm}$. Using the bulk and annular region parameters so obtained, the flow curves for other diameters are predicted and are in good agreement with the experimental data.

Thus, the network model now shows diameter-dependent flow curves because of the consideration of two different domains. This can be easily shown as follows. The total flow rate can be written as the sum of contributions from the velocity in the bulk region and in the annular region:

$$
Q_{T}=2 \pi \int_{0}^{r-\delta} r\left(v-v_{\mathrm{b}}\right) \mathrm{d} r+\pi R^{2} v_{\mathrm{b}}
$$

where $v_{\mathrm{b}}$ is velocity at the boundary of bulk and wall domain and $\delta$ the thickness of the annulus. It can 


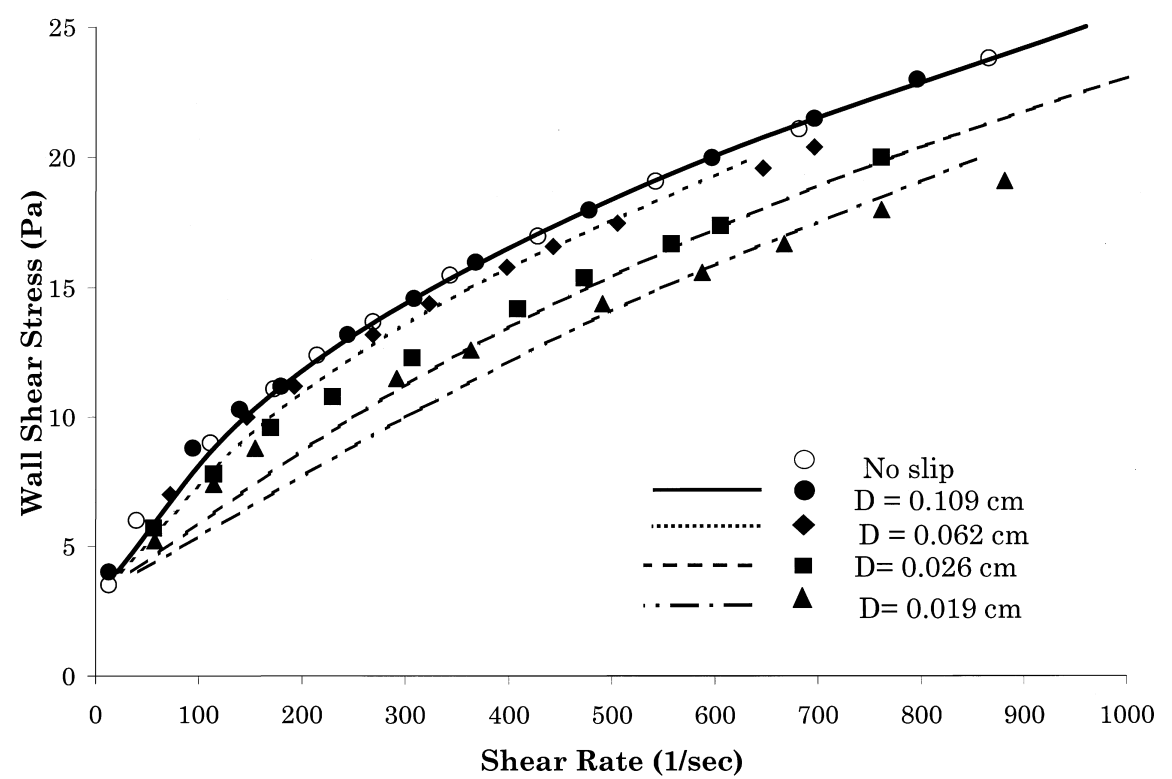

Fig. 6. Wall shear stress vs. apparent shear rate plot for transient network model using Eqs. (26) and (27). Points represent experimental data [8] and line represents model prediction. Model parameters used for the bulk are the same as given in Fig. 3. Wall parameters $F_{f}=50000, F_{g}=38000, \theta_{f}=20$, and $\theta_{g}=15.5$ are used to fit the flow curve for $D=0.109 \mathrm{~cm}$. Flow curves for other diameters are also predicted.

be shown that

$$
\dot{\gamma}_{\mathrm{a}}=\frac{4 Q_{T}}{\pi R^{3}} \equiv \frac{4 Q_{\mathrm{B}}}{\pi R^{3}}+\frac{4 v_{\mathrm{b}}}{R},
$$

where $Q_{\mathrm{B}}=2 \pi \int_{0}^{r-\delta} r\left(v-v_{\mathrm{b}}\right) \mathrm{d} r$ and $v_{\mathrm{b}} / R$ can be calculated as

$$
\frac{v_{\mathrm{b}}}{R}=\int_{1}^{1-\delta / R} \frac{g \gamma_{e}}{\lambda} \mathrm{d} r^{*} .
$$

At constant wall shear stress, the integrand is a function of $r^{*}$ only; hence, as $R$ increases, $1-\delta / R$ increases and tends to 1 at large $R$. Consequently, change in $v_{\mathrm{b}} / R$, with increase in $R$ decreases and tends to zero. Thus, the model predicts significant diameter dependence for small diameter capillaries and as the diameter increases, the flow curves gradually become diameter-independent.

Flow enhancement and diameter dependence are only indirect evidences for slip. Comparison of the predicted velocity profiles with experimentally measured velocity profiles should provide a better test for the model. As an example of direct slip measurement for polymer solution, we compare our model calculations with the experimental data of Muller-Mohnssen et al. [11] on the velocity profile of a $0.25 \%$ aqueous PAm solution. $C^{*}$ for this solution was found out to be $0.078 \%$ using an estimation similar to that discussed earlier. The model parameters for bulk flow are obtained by fitting viscosityshear rate data as shown in Fig. 7. Model parameters in the annular region are fitted so as to predict the 


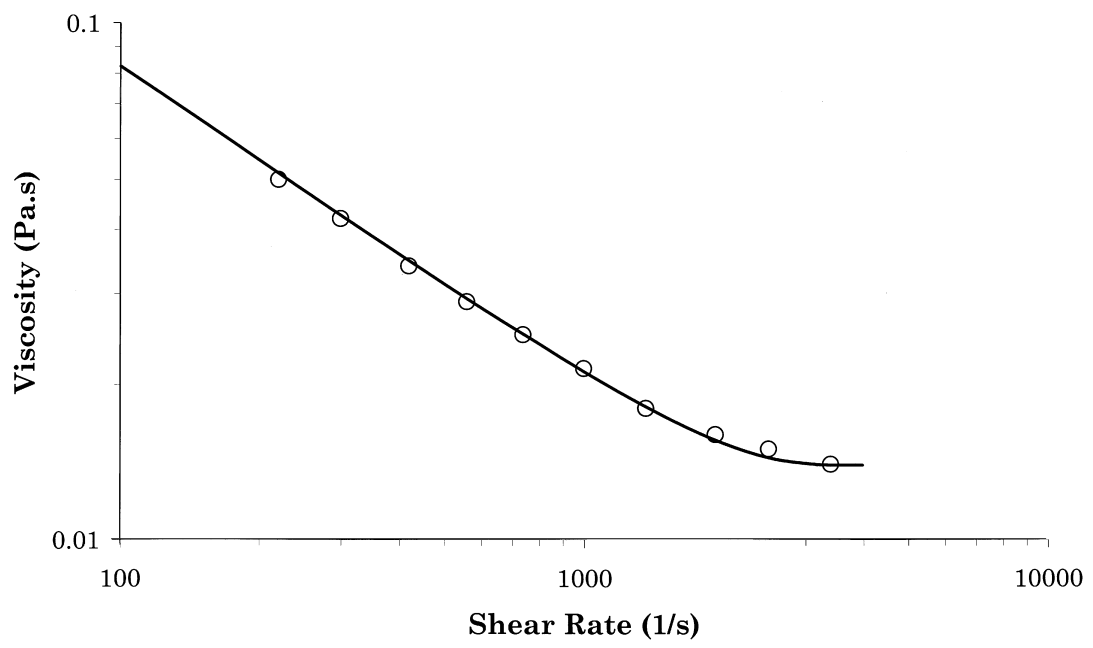

Fig. 7. Fit for experimental viscosity-shear rate data [11] using transient network model (Eqs. (26) and (27)). Fitted model parameters are $G_{0}=0.77, \lambda=8.5, F_{f}=90, F_{g}=65, \theta_{f}=12.65$ and $\theta_{g}=12.65$. Points represent the experimental data and the line represents the model fit.

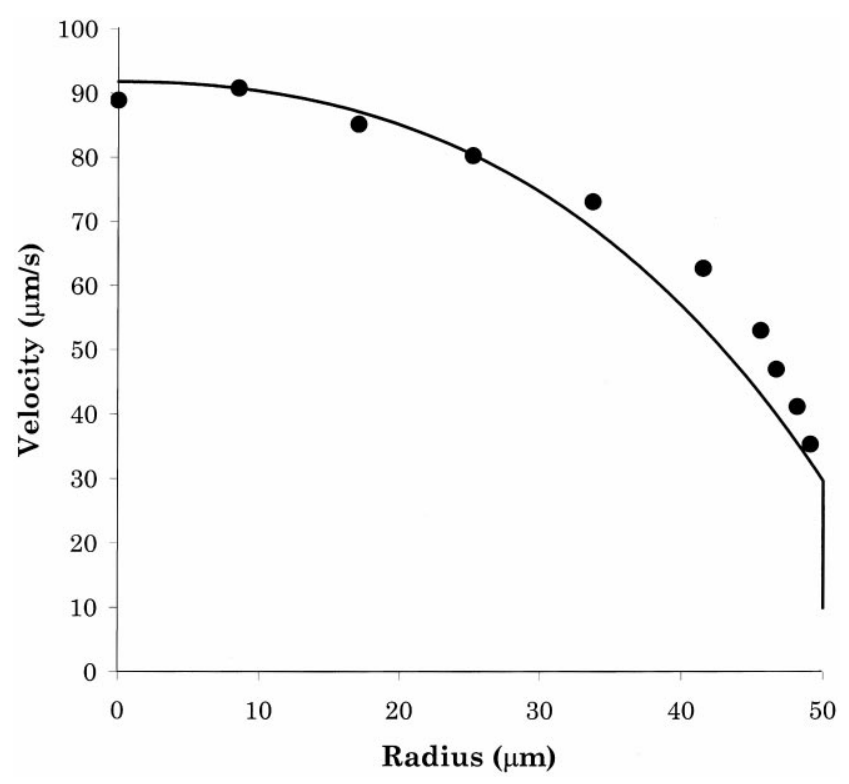

Fig. 8. Comparison of the velocity profile calculated by our model with the measured velocity profile [11]. Bulk parameters are the same as those in Fig. 7. Wall parameters are $F_{f}=900, F_{g}=2000, \theta_{f}=48.99$ and $\theta_{g}=28.28$, wall shear stress is $\tau_{\mathrm{w}}=4.6 \mathrm{~Pa}$. Points represent the experimental data and line represents the model fit.

slip shown by Muller-Mohnssen et al. [11]. Fig. 8 shows good agreement between the predicted velocity profile and the experimental velocity profile. In the foregoing calculations, we have assumed the flow to occur through a capillary of equivalent diameter. Since the experimental data was for flow 
through a rectangular conduit, this might be the reason for a small difference between the model and the experimental data.

Muller-Mohnssen et al. [11] did not measured the pressure drop across the conduit and, hence, could not detect the presence or absence of any critical wall shear stress, at which a jump in flow rate occurs. Our model calculations predict a critical stress for their data. Metzner and Cohen's data [8] does not show any critical wall shear stress. This could possibly be due to the fact that their capillary surface was pre-treated to decrease the adsorption of PAm chains on the wall. We have seen earlier that a reduction in the grafting density can reduce the critical wall shear stress. For their data, it is possible that the critical stress was below the investigated range. It is also possible that a critical stress is completely absent. As discussed earlier, a multi-valued stress is not a necessary condition for flow enhancement. In our model calculations for Metzner and Cohen's data [8], the model parameters do not predict a multivalued stress function (Eq. (28)). Our model parameters indicate that the dynamics of chains at the wall differs from that of chains in the bulk at a low wall shear stress. This is enough to predict flow enhancement and diameter-dependent flow curves shown in Figs. 5 and 4.

\subsection{Polymer melts}

Our network model can also be applied to data for an entangled polymer melt. Polymer melts are known to show a sudden enhancement in flow rate above a critical pressure drop in controlled stress capillary flow. Flow curves for melts also show diameter dependence and stick-slip oscillations in controlled flow rate capillary flow.

Fig. 9 shows the comparison of our model with the capillary flow data of Wang and Drda [29]. Apparent shear rate (without correction) is plotted against wall shear stress for capillaries of different diameters. The model is fitted for $D=1.04 \mathrm{~mm}$ and flow rates for the lower diameter capillaries are predicted. Experimental data for a polyethylene melt shows a jump in apparent shear rate (or flow rate)

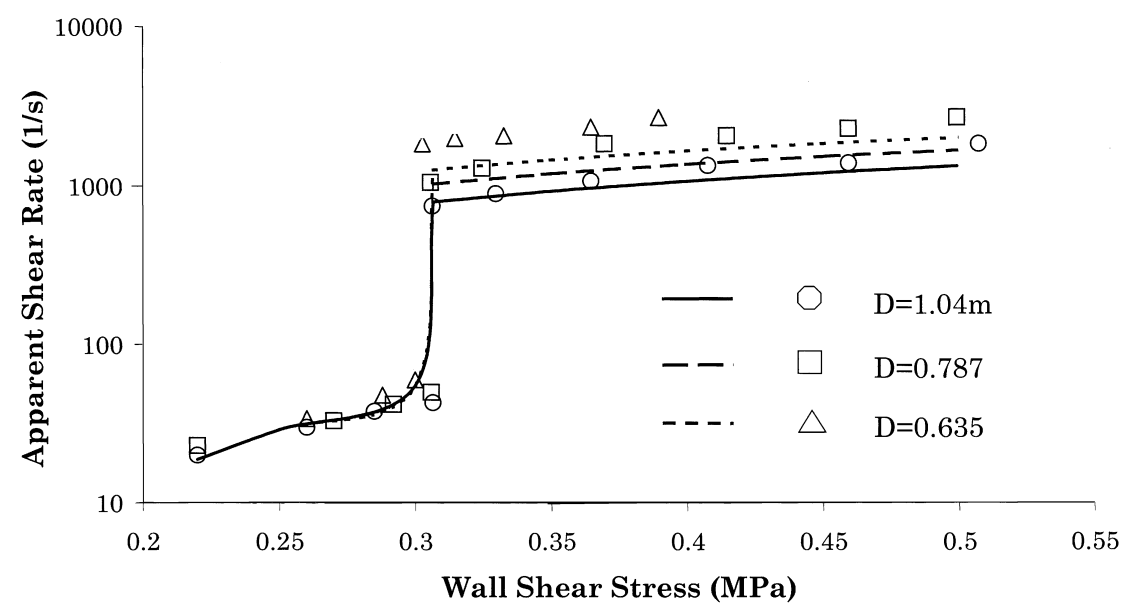

Fig. 9. Comparison of model prediction for apparent shear rate vs. wall shear stress with polyethylene melt experimental data [3]. Flow curve for $D=1.04 \mathrm{~mm}$ is fitted using model parameters $F_{f}=75, F_{g}=100, \theta_{f}=1265, \theta_{g}=12.65$ in the bulk region, and $F_{f}=60000, F_{g}=375000, \theta_{f}=178.88$ and $\theta_{g}=112.42$ in the wall region and $G_{0}=19200, \lambda=8.5$. Flow curves for other diameters are predictions. Points represent the experimental data and line represents the model fit. 


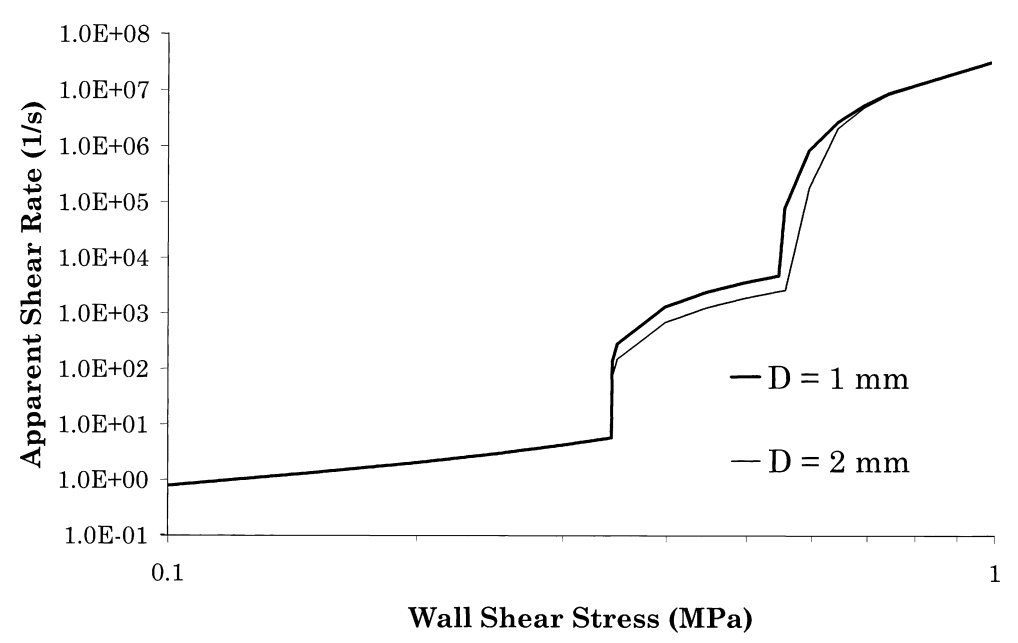

Fig. 10. Prediction of two discontinuous flow rate transitions and their diameter dependence.

at a critical shear stress of about $0.3 \mathrm{MPa}$. It can be seen that the magnitude of the jump increases with a decrease in diameter. The network model provides a good fit to the experimental data.

It is interesting to note that at very high shear stress some polymers show a second first-order transition in apparent shear rate. For example, Wang and Drda [3] show a second criticality for LLDPE resin. They found that unlike the first jump in $\dot{\gamma}_{a}$, the second jump does not show diameter-dependent flow curves. Wang and Drda argue that the second criticality in flow rate may be due to stretching of the bulk chains. Our model is able to predict bulk disentanglement at a much higher shear stress and, hence, a second jump in flow rates as shown in Fig. 10. Interestingly, our model also predicts diameterindependent flow curves after the second criticality. This is so because near the disentanglement of the bulk chains, the difference between the wall and the bulk chain dynamics is lost. Hence the capillary is now a single domain, which is responsible for the diameter-independent flow curves. However, the chain desorption models suggest that pressure dependence of viscosity can effectively cancel out the diameter dependence of flow curves [73]. Hence, it is difficult to say whether the diameter independence observed by Wang and Drda [3] in the second flow-rate jump is necessarily due to bulk disentanglement. The second criticality can arise from either bulk stretching or desorption of chains attached to the wall. We plan to investigate this phenomenon in our future work.

It is particularly interesting to compare the predicted slip length from our model with that of the Brochard-de Gennes model. An experimental study of Leger et al. [26] showed three distinct regions of slip in agreement with Brochard-de Gennes [20] model. These are:

(i) A linear friction regime at low shear rates, wherein the slip length is very small and constant with respect to slip velocity.

(ii) A non-linear friction regime above critical velocity, in which a near-linear relationship (of slope unity in a log-log plot) exists between slip length and slip velocity.

(iii) A linear friction regime at large shear rates, wherein the slip length is much larger than the size of a surface-anchored polymer molecule. 


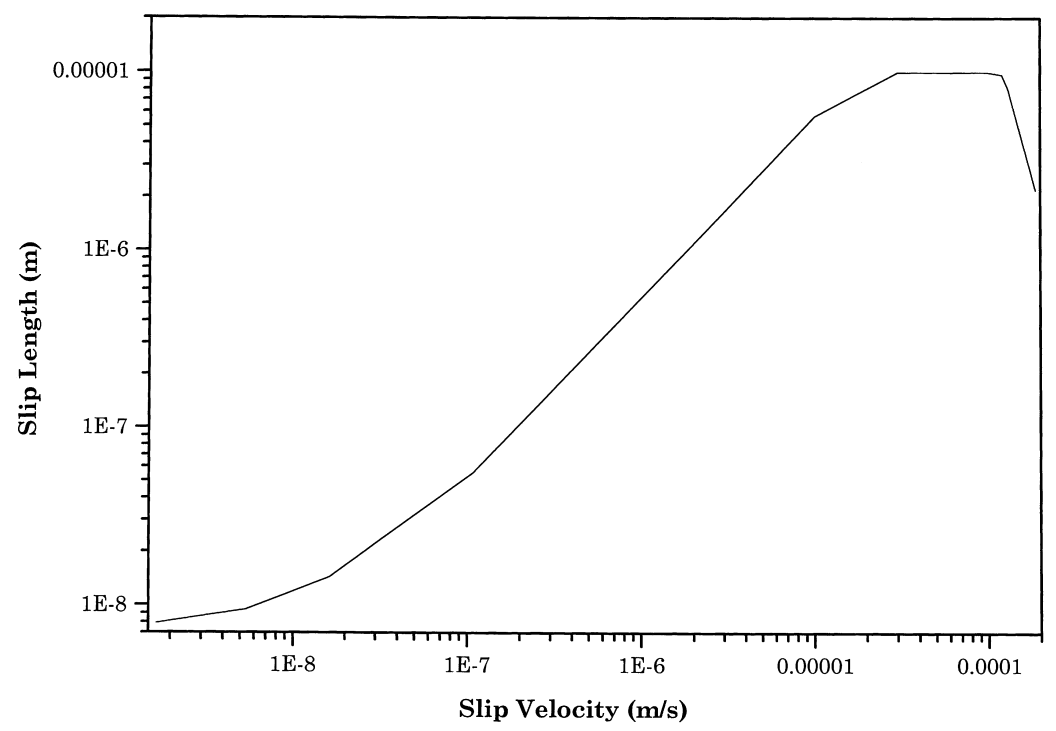

Fig. 11. Slip-length vs. slip-velocity plot for model parameters the same as in Fig. 8.

Our model predictions are shown in Figs. 11 and 12. The model successfully predicts these three regimes mentioned here. The slope of the $b$ vs. $v_{\mathrm{s}}$ curve in the second regime is predicted to be unity, which is close to that obtained by Leger et al. [26]. Here, the slip length is calculated as $b=v_{\mathrm{b}} /(\mathrm{d} v / \mathrm{d} r)$, where $v_{\mathrm{b}}$ is the boundary velocity and $\mathrm{d} v / \mathrm{d} r$ is the velocity gradient in the bulk region at the boundary. It can be seen from Fig. 11 that there exists a fourth regime in which the slip length decreases with slip

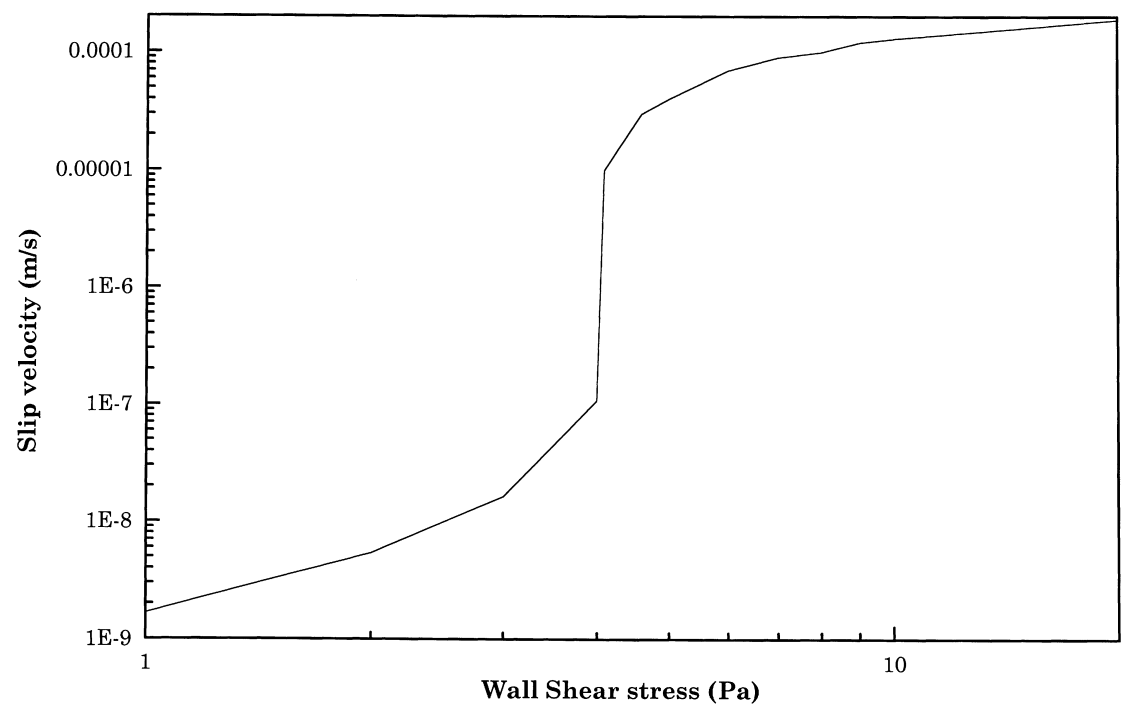

Fig. 12. Slip-velocity vs. wall shear stress plot for model parameters the same as in Fig. 8. 
velocity. This decrease in slip length is due to the increasing effective strain on bulk molecules near the boundary. The velocity gradient in the bulk increases more than the corresponding increase in the slip velocity, resulting in decrease of slip length. Though Brochard and de Gennes predicted the first three regions, they did not predict the fourth region, since their model does not consider dynamics of bulk molecules. Yang et al. [77] have indeed observed a decrease in $b$ with stress. They have defined $b$ as

$$
b=\frac{\eta_{\mathrm{B}}}{\eta_{\mathrm{I}}} a
$$

where $\eta_{\mathrm{B}}$ is the bulk viscosity, $\eta_{\mathrm{I}}$ the interfacial viscosity and ' $a$ ' the monomer length scale. They argue that, at higher stresses, $\eta_{\mathrm{B}}$ decreases due to the shear thinning nature while $\eta_{\mathrm{I}}$ remains constant (of the order of monomer viscosity after slip), which results in a decrease in $b$. Recently, Mhetar and Archer [78] have also seen a decrease in the slip length at higher shear stress in couette flow of polystyrene solution in diethyl phthalate. They interpreted this decrease to be a consequence of shear thinning, which can be related to bulk stretching and/or disentanglement.

\section{Conclusions}

We have attempted to unify various features of the slip phenomenon in one theoretical framework. Unification has been achieved for systems (solutions and melts) and for the underlying physical mechanisms (wall disentanglement, desorption and bulk disentanglement).

We have modeled wall-slip by using a transient network model, in which a dynamic network near the wall is formed by entanglements between adsorbed chains and bulk chains. The network can be broken by either disentanglement of chains or by desorption of the wall chains. We have considered only the first mechanism in this work. We show that, the model predicts flow rate enhancement, diameterdependent flow curves, decrease in diameter dependence with increase in radius, a discontinuous jump in flow rate for controlled pressure-drop experiments and a second jump in flow rate at a higher stress. The model predicts a non-monotonic flow curve for severe disentanglement. The model also predicts three different regimes for the slip-length/slip velocity relation. Further, the critical stress is predicted to depend directly on the grafting density of adsorbed chains and also on temperature (provided no desorption occurs).

\section{List of symbols}

a monomer length scale (Eq. (36))

$a, b \quad$ arbitrary constants

$C^{*} \quad$ critical (overlap) concentration

$D \quad$ diameter of capillary

$F_{f}, F_{g} \quad$ empirical parameters in Eqs. (26) and (27)

$F_{\mathrm{H}} \quad$ force of desorption

$F_{\mathrm{T}} \quad$ tension in freely joined chain

$f \quad$ rate of creation 


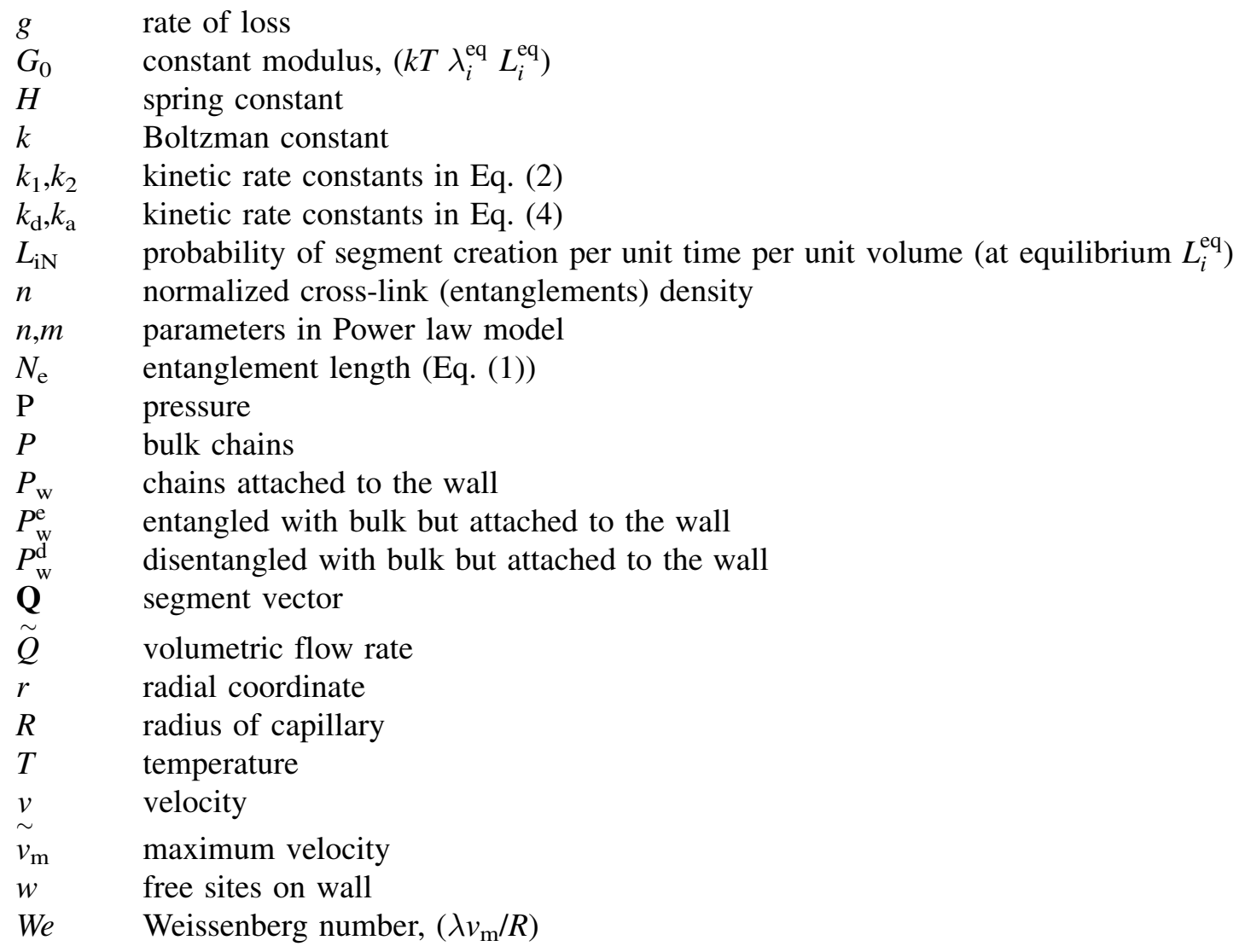

\section{Greek letters}

$\alpha_{f}, \alpha_{g} \quad$ empirical parameters in Eqs. (26) and (27)

$\delta \quad$ unit tensor

$\approx \quad$ thickness of wall layer

$\phi \quad$ fraction of surface coverage of molecules attached to the wall

$\varphi$ fraction of surface coverage of molecules entangled with the bulk and attached to the wall

$\gamma_{\mathrm{e}} \quad$ effective strain, $\left\{\left(\tau_{11}-\tau_{22}\right) / 2 \tau_{12}\right\}$

$\underset{\sim}{\dot{\gamma}} \quad$ rate of strain tensor $\left(\underset{\sim}{\nabla} \underset{\sim}{\boldsymbol{v}}+(\underset{\sim}{\nabla} \underset{\sim}{\boldsymbol{v}})^{T}\right.$

$\approx \quad$ viscosity

$\eta_{\mathrm{B}} \quad$ bulk viscosity (Eq. (36))

$\eta_{\mathrm{I}} \quad$ interfacial viscosity (Eq. (36))

$\underset{\sim}{\kappa} \quad$ transpose of deformation gradient tensor, $(\underset{\sim}{\nabla v})^{T}$

$\widetilde{\lambda}_{\mathrm{iN}} \quad$ probability of segment loss per unit time per unit volume. (at equilibrium $\lambda_{i}^{\mathrm{eq}}$ )

$\lambda_{\mathrm{i}} \quad$ relaxation time 


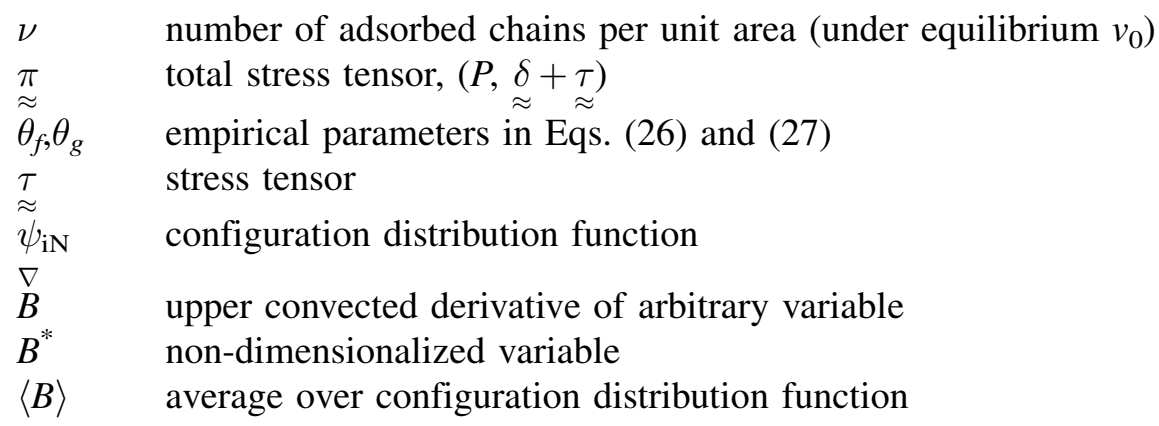

\section{Acronyms}

HDPE high density polyethylene

LDPE low density polyethylene

LLDPE linear low density polyethylene

PAm polyacrylamide

PDMS polydimethylsiloxane

PEO poly ethylene oxide

PS polystyrene

PMMA poly methyl metha acrylate

PVC polyvinylchloride 


\section{Appendix A}

\section{Experimental observations on wall slip in polymer solutions}

\begin{tabular}{lll}
\hline Reference & Polymer solution & Experimental method and observations \\
\hline$[48]$ & PMMA in monochlorobenzene & $\begin{array}{l}\text { Noticeable slip effect for concentration } \\
\text { above } 2.5 \mathrm{~g} / \mathrm{l}\end{array}$ \\
{$[49]$} & PEO in water & $\begin{array}{l}\text { Apparent viscosity decreases with } \\
\text { capillary diameter }\end{array}$
\end{tabular}

[50] Aqueous solution of PVOH and sodium borate

$$
\text { O.5\% aqueous PAM }
$$

$$
0.25 \% \text { aqueous PAM }
$$

$$
\text { HEC in water }
$$

$0.6 \%$ aq. PAM; $0.4 \%$ aq. Xanthan gum

\section{$[52,53] \quad$ PAM in water}

Xanthan in water

Polystyrene solution

PAM, PEO
Capillary flow in rough and smooth tubes. Slip measured by hot-film anemometer Extrudate distortion observed in rough tube at lower apparent shear rate.

Flow measurements. Flow rate enhancement and diameter dependence

Measured velocity profile using tracer particles. Observed high velocity gradient near wall. Observed a decrease in slip velocity if $\mathrm{Ca}^{++}$ and $\mathrm{Na}^{+}$were added to solution. Slip velocity function of wall shear stress, polymer concentration and capillary diameter.

Flow measurements. Flow rate enhancement and diameter

dependence. Greater slip for rodlike xanthan than for PAM

Apparent viscosity decreases with decrease in diameter

Apparent viscosity decreases with decrease in diameter

Displacement of tracer particles in plane couette

Flow curves show diameter independence but $\mathrm{L} / \mathrm{D}$ ratio dependence, which become asymptotic as L/D ratio increases
Remarks

Anomalous behavior near wall observed above $5 \mathrm{gm} / \mathrm{l}$

Different solutions of various molecular weights indicate a relation between friction reduction and volumetric concentration

The microscopic nature of the wall can promote or inhibit macroscopic slip. Slip at the wall reduces extrudate swell and delays the onset of extrusion instabilities. Slip decreases ocal momentum transfer and increases local heat transfer.

Results are in qualitative agreement with proposed diffusion theory (stress-induced migration)

Infers that $0.1 \mu \mathrm{m}$ thickness layer near wall behaves like a highly dilute solution of low viscosity. Infers stress-induced migration of chains away from wall

Surface characteristics undergo a dramatic change from Polymer adsorption gel formation at the tube surface to the phenomena characterised by slip in narrow tube

Infers that effect of polymer -wall interaction influences molecular conformation in flow and the corresponding formation of anisotropic layer

Depletion thickness near wall was close to radius of gyration and decreases rapidly with concentration Flow in long capillaries is divided in to four regions, entrance, developing flow, developed flow and exit Inferred that slip is observed either at wall or at thin disentangling layer near wall

Proposed a new method for correction of end effects 
Appendix A (Continued)

\begin{tabular}{|c|c|c|c|}
\hline Reference & Polymer solution & Experimental method and observations & Remarks \\
\hline [56] & Xanthan solution & $\begin{array}{l}\text { Flow measurements in glass } \\
\text { capillaries. Temperature, concentration } \\
\text { and diameter dependence studied }\end{array}$ & $\begin{array}{l}\text { Slip velocity was found to be dependent on sample, } \\
\text { concentration and independent of temperature }\end{array}$ \\
\hline [57] & $0.2 \%$ aqueous xanthan solution & $\begin{array}{l}\text { Apparent slip detected by Rheo-NMR. } \\
\text { No slip detected after degradation }\end{array}$ & $\begin{array}{l}\text { Slip mechanism may be associated with the } \\
\text { formation of gel-like structures in the } \\
\text { xanthan solution }\end{array}$ \\
\hline [34] & Polystyrene solutions & $\begin{array}{l}\text { Couette flow. Significant level of slip } \\
\text { seen during steady shearing of } \\
\text { entangled polystyrene solution }\end{array}$ & $\begin{array}{l}\text { The slip is in qualitative agreement with predictions } \\
\text { of Brochard and de Gennes; slip length is much } \\
\text { greater than expected for entangled system }\end{array}$ \\
\hline$[5,33]$ & $\begin{array}{l}\text { Polystyrene in tricresyl phosphate and } \\
\text { in diethyl phthalate }\end{array}$ & $\begin{array}{l}\text { A critical stress is observed above which } \\
\text { slip is seen. Critical stress is independent } \\
\text { of solvent and molecular weight }\end{array}$ & $\begin{array}{l}\text { Inferred that origin of slip is due to disentanglement } \\
\text { of bulk molecules with those attached to wall rather } \\
\text { than desorption of molecules attached to the wall }\end{array}$ \\
\hline [78] & PS in diethyl phthalate & $\begin{array}{l}\text { Couette shear flow. Observed decrease in } \\
\text { slip length at very high shear stress }\end{array}$ & $\begin{array}{l}\text { Slip behavior was observed to be a strong function of } \\
\text { nature of surface. Decrease in slip length at high } \\
\text { stress is attributed to shear thinning }\end{array}$ \\
\hline
\end{tabular}


Appendix B

Various theoretical analyses on slip for polymer solutions and melts

\begin{tabular}{lll}
\hline Reference Approach & Prediction \\
\hline
\end{tabular}

Polymer solutions

$[12]$

[13]

[14]
Stress induced migration: thermodynamic theory

Stress induced migration: fluid mech. Theory

Polymer species dissolved in the fluid diffuse away from the wall, which generates a region of low viscosity adjacent to wall and as a result bulk slips

Stress induced migration: two fluid approach (based on Osaki, Doi theory, 1991)

\section{Constitutive instability}

Constitutive instability

Junctions are assumed between wall/polymer interface as well as in the bulk of the polymer fluid. Junctions are

described by kinetic equation describing a reaction between bonded and free macromolecules at the interface Viscoelastic fluid model is proposed which exhibits local maxima of steady state shear. Suggests that there may

be phenomena which can give appearance of slip but which are governed solely by material properties of fluid Peeling experiments on LLDPE are shown to predict onset of surface distortion and functional form and magnitude of subsequent wall slip

Before critical wall shear stress there exists a no slip boundary condition. When such critical stress is

exceeded molecules attached to the wall undergo coil to stretch transition and bulk molecules slip by

disentanglement from attached molecules

The molecular model for slip of a polymer as it flows throug a die is based on the concept of the activation rate theory of the kinetics of chemical reactions

Nonlinear constitutive equation (K-BKZ) is introduced into relaxation-oscillation model

A multi-mode interfacial constitutive equation for molten polymers. Hookean segments with different relaxation times were assumed
Migration towards center

No radial migration

Concentration profile develops down stream from the tube entry. Only small decrease in concentration of polymer near wall can give rise to significant slip

No radial migration

Many-valued shear stress

Predicts hysteresis loop

Prediction of the temperature dependence of wall-slip

velocities. At constant temperature, slip velocity depends on wall shear stress according to a power law

Experimental data on spurt is fitted.

Critical stress is relatively insensitive to temperature. Flow curves are diameter independent due to pressure dependence of viscosity

Critical stress at which slip occurs is directly proportional to the temperature of the melt and density of the grafted chains

The model correctly predicts the dependence of slip velocity of high molecular weight, linear polyethylene resins on temperature, pressure, wall shear stress, and molecular weight Non-monotonicity of the stress-strain curve is observed.

A boundary layer is developed which shows oscillatory behavior Model predicts steady state slip data quantitatively and dynamic slip data qualitatively 
Appendix B (Continued)

\begin{tabular}{lll}
\hline Reference & Approach & Prediction \\
\hline$[61]$ & $\begin{array}{l}\text { The model consists of a viscoelastic constitutive equation } \\
\text { for polymer flows in bulk, the wall-slip model and model }\end{array}$ & $\begin{array}{l}\text { It describes onset of critical conditions for spurt, hysteresis, } \\
\text { and pressure oscillations }\end{array}$
\end{tabular}
and pressure oscillations

[62]

for compressibility effect in capillary flows

multiplicity is studied using a multivalued slip relation to model the phenomena of the hysteresis and spurt

[19] A chemical theory is developed by modelling the exchange of bridging sites between two opposing polymeric and solid surfaces. A catastrophic loss of adhesion occurs at a critical stress, which depends on difference in work of adhesion (polymer-polymer) and work of cohesion Multiplicity of the curve is seen to be absent for high $L / D$ ratio (polymer-wall)

The polymer slips at all stresses. Slip velocity obeys time free volume superposition and depends on both shear and normal stresses 
Appendix C

Experimental observations of wall slip in extrusion of melts

\begin{tabular}{lll}
\hline Reference & Polymer melt & Observations \\
\hline$[80]$ & PE & Capillary flow. Hysteresis is observed
\end{tabular}

Remarks

There are three modes of flow. One related to smooth extrudate. Other related to rough extrudate, and last related to spiral filaments

[81] PE and ethylene-propylene copolymer

HDPE

PE

Elastic properties of polyethylene melts at high shear rates with respect to extrusion

Filled PP and PA 66

Range of rubbers

[66] Slip flow of non-platicized PVC compounds

\section{HDPE LLDPE melt}

Various polyethylene
Capillary flow. Observed that hysteresis loop becomes shallower as polydispersity increases

Shape of extrudate varies periodically with time, sharkskin plug and rough surface

Short time sandwich rheometer.

Stick-slip transition and extrudate

distortion is seen. Critical shear rate

for high mol. wt. is lower than that of low mol. wt.

Injection capillary flow with various capillaries in series. Nonmonotonic pressure/flow rate curves can be seen for multiple capillaries in series

Slip with pressure oscillations seen for HDPE. Slip is not observed for LDPE

Shows presence of a critical stress at which flow rate increases discontinuously

Slit rheometer. Wall slip only occurred for compounds with very high shear viscosity, which corresponds to high mol. wt

Stick-slip behavior seen in melt

Critical stress is relatively insensitive to molecular characteristics. Melt fracture is strong function of material of construction of die
Occurrence of oscillatory flow can be avoided

by choice of a resin with proper balance

of mol. wt. and polydispersity

Sudden increase in flow rate is believed to arise from multivalued flow curve

Shear rate dependence of shear stress

is correctly described on the basis of

discrete relaxation time spectrum

Mechanism of unsteady flow is investigated by fiber orientation using molding simulation

It is inferred that the molecular mechanism responsible for the pressure oscillation within a certain range of deformation rates must

be based on the inability of the polymer melt to sustain larger strain than critical strain

The spurting and sliding flow is characterized by transition from a fluid to a highly

elastic state

Concludes that energy dissipated during flow is not decisive for shear or slip flow. For a given output, power dissipated is found to be lower than that for wall adhesion

Inferred that slip is due to failure of adhesion between metal-polymer interface

Inferred that slip is due to failure of adhesion 


\begin{tabular}{ll}
\hline Reference & Polymer melt \\
\hline$[67]$ & HDPE through rectangular conduit \\
{$[68]$} & Several PBs \\
{$[6]$} & HDPE \\
{$[69]$} & SBR compound \\
{$[70]$} & LLDPE \\
& $\begin{array}{l}\text { Polydimethylsiloxane, polybutadiene } \\
\text { and polyethylene }\end{array}$ \\
{$[71]$} & PDMS
\end{tabular}

Monodispersed PS

PE

\section{LLDPE}

Observations

Measurements by hot film probe

\section{Slip phenomenon probed by hot-film} anemometer technique

Slip is observed above critical stress. Slip velocity depends on $L / D$ ratio Found a power-law relationship between wall slip, wall shear stress and geometry of the flow

Found a stable window in stick-slip region with no oscillations and low pressure and undistorted surface

Slip is observed at a critical stress

For weak grafting density surface, a sharp transition between no-slip and slip condition is seen

The slip velocity is proportional to the shear rate in the Newtonian flow regime considered and the proportionality constant is related to the mol. wt. to the $1.11 \pm 0.33$ power Show a temperature window of least flow resistance, after which pressure oscillations are seen

Showed first order transition in flow rate at a critical shear stress. Also showed diameter and temperature dependence. Observed two critical stresses

Experiments performed using various die materials, which show different critical shear rates. In addition, gap dependence is not seen

Critical shear stress (where instability began) was found to be much less than conventional values
Remarks

Under slip conditions, relation between $\mathrm{Nu}$ and

Pe departed from that excepted under no slip Lowest molecular wt. PB amongst four different samples of PB did not show slip while others showed pressure oscillations and slip Melt slip is a result of an adhesive failure occurring at the wall-polymer interface Mooney method failed to fit the experimental data

Mesoscopic transition has been suggested for such a pressure decrease

Slip behavior explained with a model having static friction stress at wall

Shear induced elongation of adsorbed chains is responsible for slip

Various apparent slip models were presented and were argued to be invalid, thus concluded that the true slip was presen

In situ X-ray experiments show existence of hexagonal phase

Infers that chain stretching may be the right mechanism for slip on unmodified surfaces and chain desorption is the mechanism for modified surface

The flow curves can be fitted with the slip theory of Hill et al. [17]

Infers that slip is dominated by competition between flow induced chain detachment and disentanglement of the chains adsorbed at wall 
Appendix C (Continued)

\begin{tabular}{|c|c|c|c|}
\hline Reference & Polymer melt & Observations & Remarks \\
\hline [75] & Linear and branched PE & $\begin{array}{l}\text { Extrudate distortion and pressure } \\
\text { oscillations observed }\end{array}$ & $\begin{array}{l}\text { Inferred that these do not arise from slip } \\
\text { but from melt compressibility }\end{array}$ \\
\hline [76] & Polyethylene & $\begin{array}{l}\text { Sharkskin effects observed by profilometry, } \\
\text { optical microscopy and observation of } \\
\text { cross section }\end{array}$ & $\begin{array}{l}\text { Resins exhibiting long chain branching and } \\
\text { strain hardening are less sensitive to sharkskin. } \\
\text { An increase in temperature is shown to shift } \\
\text { the onset and the development of the } \\
\text { sharkskin to higher shear rates }\end{array}$ \\
\hline [77] & PS, LDPE, EVA & $\begin{array}{l}\text { Observed no slip and pressure oscillations in } \\
\text { bare aluminum dies and observed slip when } \\
\text { wall is coated with fluoropolymer. ' } b \text { ' found } \\
\text { to decrease with stress at higher stress }\end{array}$ & $\begin{array}{l}\text { Absence of slip in Bare } \mathrm{Al} \text { die is inferred from } \\
\text { Mooney analysis, decrease in ' } b \text { ' is due to } \\
\text { shear thinning }\end{array}$ \\
\hline
\end{tabular}




\section{Appendix D}

\section{A. Derivation of Eq. (28) from constitutive equation}

The constitutive equation for transient network model is given as

$$
g \underset{\approx}{\tau^{*}+W e} \underset{\approx}{\stackrel{\nabla}{\tau}} \underset{\approx}{\approx}=-W e \underset{\approx}{\gamma^{*}}-(f-g) \underset{\approx}{\delta} .
$$

For shear flow we have, $\underset{\sim}{v}=\underset{\sim}{v}\left(v_{1}\right), v_{1}=v_{1}\left(x_{2}\right)$,

$$
\underset{\approx}{\tau}=\underset{\approx}{\tau}(r)=\left(\begin{array}{lll}
\tau_{11} & \tau_{12} & 0 \\
\tau_{21} & \tau_{22} & 0 \\
0 & 0 & \tau_{33}
\end{array}\right) \quad \underset{\approx}{\underset{\gamma}{\gamma}=\frac{\mathrm{d} v_{1}}{\mathrm{~d} x_{2}}}\left(\begin{array}{ccc}
0 & 1 & 0 \\
1 & 0 & 0 \\
0 & 0 & 0
\end{array}\right) \text { and }
$$

Hence, we get

$$
\begin{aligned}
& g \tau_{22}^{*}=-f+g \\
& g \tau_{33}^{*}=-f+g \\
& g \tau_{11}^{*}-2 W e \tau_{12}^{*} \frac{\partial v_{1}^{*}}{\partial x_{2}^{*}}=-f+g \\
& g \tau_{12}^{*}-W e \tau_{22}^{*} \frac{\partial v_{1}^{*}}{\partial x_{2}^{*}}=-W e \frac{\partial v_{1}^{*}}{\partial x_{2}^{*}} .
\end{aligned}
$$

From Eqs. (2) and (3) it can be seen that $\tau_{22}^{*} \equiv \tau_{33}^{*}=(-f+g) / g$. Inserting this in Eq. (4) we get

$$
W e \frac{\partial v_{1}^{*}}{\partial x_{2}^{*}}=\frac{\tau_{11}^{*}-\tau_{22}^{*}}{2 \tau_{12}^{*}} g
$$

which can be written as

$$
\frac{\partial v_{1}^{*}}{\partial x_{2}^{*}}=\frac{\gamma_{e} g}{W e}
$$

Now using Eqs. (5) and (7), we get

$$
\tau_{12}^{*}=\gamma_{\mathrm{e}} \frac{f}{g}
$$

Eqs. (7) and (8) give a final set of constitutive equations to be solved.

\section{References}

[1] D.S. Kalika, M.M. Denn, Wall slip and extrudate distortion in linear low-density polyethylene, J. Rheol. 31 (1987) 815834. 
[2] N. El. Kissi, J.M. Piau, The different capillary flow regimes of entangled polydimethylsiloxane polymers: macroscopic slip at wall, hysteresis and cork flow, J. Non-Newtonian Fluid Mech. 37 (1990) 55-94.

[3] S-Q. Wang, P.A. Drda, Molecular instabilities in capillary flow of polymer melts: interfacial stick-slip transition, wall slip and extrudate distortion, Macromol. Chem. Phys. 198 (1997) 673-701.

[4] K.B. Migler, G. Massey, H. Hervet, L. Leger, The slip transition at polymer-solid interface, J. Phys. Condens. Matter 6 (1994) A301-A304.

[5] M.J. Reimers, J.M. Dealy, Sliding plate rheometer studies of concentrated polystyrene solutions: large amplitude oscillatory shear of a very high molecular weight polymer in diethyl phthalate, J. Rheol. 40 (1996) 167-186.

[6] S.G. Hatzikiriakos, J.M. Dealy, Wall slip of molten high-density polyethylene. I. Sliding plate rheometer studies, J. Rheol. 35 (1991) 497-523.

[7] A. Dutta, R.A. Mashelkar, On the slip effect in free coating of non-Newtonian fluids, Rheol. Acta. 21 (1982) 52-61.

[8] Y. Cohen, A.B. Metzner, Slip phenomena in polymer solutions flowing through small channels, AICHE Symp. Ser No. 212, 78 (1982) 77-85.

[9] L. De Vargas, O. Manero, On the slip phenomenon of polymeric solutions through capillaries, Polym. Engng. Sci. 29 (1989) 1232-1236.

[10] J.W.H. Kolnaar, A. Keller, A singularity in the melt flow of polyethylene with wider implications for polymer flow rheology, J. Non-Newtonian Fluid Mech. 69 (1997) 71-98.

[11] H. Muller-Mohnssen, H.P. Lobl, W. Schauerte, Direct determination of apparent slip for a ducted flow of polyacrylamide solutions, J. Rheol. 31 (1987) 323-336.

[12] M. Tirrel, M.F. Malone, Stress induced diffusion of macromolecules, J. Polym. Sci. Polym. Phys. Ed. 15 (1977) 15691583.

[13] J.H. Aubert, M. Tirrell, Macromolecules in non-homogeneous velocity gradient fields, J. Chem. Phys. 72 (1980) 26942700.

[14] T.W. Huseby, Hypothesis on a certain flow instability in polymer melts, Trans. Soc. Rheol. 10 (1966) 181-190.

[15] T.C.B. McLeish, Stability of the interface between two dynamic phases in capillary flow of linear polymer melts, J. Polym. Sci.: Part B: Polym. Phys. 25 (1987) 2253-2264.

[16] R.W. Kolakka, D.S. Malkus, M.G. Hansen, G.R. Ierley, R.A. Worthing, Spurt phenomena of the Johnson-Segalman fluid and related models, J. Non-Newtonian Fluid Mech. 29 (1988) 303-335.

[17] D.A. Hill, T. Hasegawa, M.M. Denn, On the apparent relation between adhesive failure and melt fracture, J. Rheol. 34 (1990) 891-918.

[18] C.W. Stewart, Wall slip in the extrusion of linear polyolefins, J. Rheol. 37 (1993) 499-513.

[19] D.A. Hill, Wall slip in polymer melts: a pseudo-chemical model, J. Rheol. 42 (1998) 581-601.

[20] F. Brochard, P.G. de Gennes, Shear-dependent slippage at a polymer/solid interface, Langmuir 8 (1992) $3033-3037$.

[21] G.V. Vinogradov, V.P. Protasov, V.E. Dreval, The rheological behavior of flexible-chain polymers in the region of high shear rates and stresses, the critical rate and process of spurting, and supercritical conditions of their movement at $T>T_{\mathrm{g}}$, Rheol. Acta 23 (1984) 46-61.

[22] A.V. Ramamurthy, Wall slip in viscous fluids and influence of materials of construction, J. Rheol. 30 (1986) $337-357$.

[23] W.R. Schowalter, The behavior of complex fluids near solid boundaries, J. Non-Newtonian Fluid Mech. 29 (1988) 2536.

[24] M.M. Denn, Issues in viscoelastic fluid mechanics, Annu. Rev. Fluid Mech. 22 (1990) 13-34.

[25] R.G. Larson, Instabilities in viscoelastic flows, Rheol. Acta 31 (1992) 213-263.

[26] L. Leger, H. Hervet, G. Massey, The role of attached polymer molecule in wall slip, Trends Poly. Sci. 5 (1997) 40-45.

[27] R.G. Larson, Constitutive Equations for Polymer Melts and Solutions, Butterworths series in Chemical Engineering, Boston, 1988, pp. 120.

[28] T.C.B. Mcleish, R.C. Ball, A molecular approach to the spurt effect in polymer melt flow, J. Polym. Sci.: Part D: Polym. Phys. 24 (1986) 1735-1745.

[29] S.-Q. Wang, P.A. Drda, Super fluid like stick-slip transition in capillary flow of linear polyethylene melt. 1. General features, Macromolecules 29 (1996) 2627-2632.

[30] J.W.H. Kolnaar, A. Keller, A temperature window of reduced flow resistance in polyethylene with implications for melt flow rheology: 1. The basic effect and principal parameters, Polymer 35 (1994) 3863-3873.

[31] H.M.M. van Bilsen, H. Fischer, J.W.H. Kolnaar, A. Keller, A temperature window of reduced flow resistance in Polyethylene: in Situ WAXS, Macromolecules 28 (1995) 8523-8527. 
[32] L.A. Archer, Y-L. Chen, R.G. Larson, Delayed slip after step strains in highly entangled polystyrene solutions, J. Rheol. 39 (1995) 519-525.

[33] M.J. Reimers, J.M. Dealy, Sliding plate rheometer studies of concentrated polystyrene solutions: nonlinear viscoelasticity and wall slip of two high molecular weight polymers in tricresyl phosphate, J. Rheol. 42 (1998) 527-548.

[34] V.R. Mhetar, L.A. Archer, Secondary flow of entangled polymer fluids in plane couette shear, J. Rheol. 40 (1996) 549572.

[35] S.G. Hatzikiriakos, N. Kalogerakis, A dynamic slip model for molten polymers based on a network kinetic theory, Rheol. Acta 33 (1994) 38-47.

[36] S.G. Hatzikiriakos, A multimode interfacial constitutive equation for molten polymers, J. Rheol. 39 (1995) 61-71.

[37] R.B. Bird, R.C. Armstrong, O. Hassager, Dynamics of Polymeric Liquids, vol. 2, Kinetic Theory, John Wiley \& Sons. New York, 1987, pp. 359.

[38] E.P. Vrahopoulou, A.J. McHugh, Theory with non-Gaussian chain segments, J. Rheol. 31 (1987) 371-384.

[39] F. Tanaka, S.F. Edwards, Viscoelastic properties of physically cross-linked networks, transient network theory, Macromolecules 25 (1992) 1516-1523.

[40] S. Wang, Transient network theory for shear thickening fluids and physically cross-linked systems, Macromolecules 25 (1992) 7003-7010.

[41] K.H. Ahn, K. Osaki, A network model predicting the shear thickening behavior of poly(vinyl alcohol)/sodium borate aqueous solution, J. Non-Newtonian Fluid Mech. 55 (1994) 215-227.

[42] K.H. Ahn, K. Osaki, Mechanism of shear thickening investigated by a network model, J. Non-Newtonian Fluid Mech. 56 (1995) 267-288.

[43] G. Akay, Unstable capillary flow of reinforced polymer melts, J. Non-Newtonian Fluid Mech. 13 (1983) 309-323.

[44] J.W.H. Kolnaar, A. Keller, A temperature window of reduced flow resistance in polythene with implications for melt flow rheology: 2. Rheological investigations in the extrusion window, Polymer 36 (1995) 821-836.

[45] Y. Cohen, The behavior of polymer solutions in non-uniform flows, Ph.D. thesis, University of Delware, Newark, 1981.

[46] W.M. Kulicke, R. Kniewske, J. Klien, Preparation characterization solution properties and rheological behavior of polyacrylamide, Prog. Polym. Sci. 8 (1982) 373-468.

[47] J. Brandrup, E.H. Immergut (Eds.), Polymer Handbook. John Wiley \& Sons. New York, 1989, p. VII/34.

[48] B.A. Toms, Detection of wall effects in laminar flow of solutions of a linear polymer, J. Coll. Sci. 4 (1949) 511.

[49] V.N. Kalashnikov, S.A. Valsov, On scale-dependent effect in laminar flow of dilute polymer solutions in tubes, Rheol. Acta 17 (1978) 296-302.

[50] A.M. Kraynik, W.R. Schowalter, Slip at the wall and extrudate roughness with aqueous solution of poly vinyl alcohol and sodium borate, J. Rheol. 25 (1981) 95-114.

[51] W. Kozicki, C.J. Hsu, S.N. Pasari, Evaluation of polymer adsorption gel formation and slip in polymer solution flows, Chem. Engng. Commun. 59 (1987) 137-160.

[52] A. Omari, M. Moan, G. Chauveteau, Wall effects in flow of flexible polymer solutions through small pores, Rheol. Acta 28 (1989) 520-526.

[53] A. Omari, M. Moan, G. Chauveteau, Hydrodynamic behavior of semirigid polymer at solid-liquid interface, J. Rheol. 33 (1989) $1-13$.

[54] J. Perez-Gonzalez, L. De Vargas, G. Tejero, Flow development of xanthan solutions in capillary rheometers, Rheol. Acta 31 (1992) 83-93.

[55] L. De Vargas, J. Pkrez-Gonzhlez, J. Romero-Barenque, Evaluation of end effects in capillary rheometers for solutions of flexible polymers, J. Rheol. 39 (1995) 125-137.

[56] M.A. Valdez, L. Yeomans, F. Montes, H. Acuna, A. Ayala, Influence of temperature on the slip velocity of semidilute xanthum gum solutions, Rheol. Acta. 34 (1995) 474-482.

[57] C.J. Rofe, L. de Varges, J. Pkrez-Gonzhlez, R.K. Lambert, P.T. Callaghan, Nuclear magnetic resonance imaging of apparent slip effects in xanthan solutions, J. Rheol. 40 (1996) 1115-1128.

[58] U.S. Agarwal, A. Dutta, R.A. Mashelkar, Migration of macromolecules under flow: The physical origin and engineering implications, Chem. Eng. Sci. 49 (1994) 1693-1717.

[59] H.C. Lau, W.R. Schowalter, A model for adhesive failure of viscoelastic fluids during flow, J. Rheol. 30 (1986) 193-206.

[60] J. Molenaar, R.J. Koopmans, Onset of sharkskin phenomena in polymer extrusion, Phys. Rev. E 58 (1998) 4683-4691.

[61] K.P. Adewale, A.I. Leonov, Modeling spurt and stress oscillations in flows of molten polymers, Rheol. Acta 36 (1997) $110-127$. 
[62] K.A. Kumar, M.D. Graham, Effect of pressure-dependent slip on flow curve multiplicity, Rheol. Acta 37 (1998) 245255.

[63] A. Weill, Capillary flow of linear polyethylene melt: sudden increase in flow rate, J. Non-Newtonian Fluid Mech. 7 (1980) 303-314.

[64] H.M. Laun, Elastic properties of polyethylene melt at high shear rates with respect to extrusion, Rheol. Acta. 21 (1982) 464-469.

[65] L.A. Utracki, R. Gendron, Pressure oscillation during extrusion of polyethylenes II, J. Rheol. 28 (1984) 601-623.

[66] W. Knappe, E. Krumbiick, Slip flow of non-plasticized PVC compound, Rheol. Acta 25 (1986) 296-307.

[67] B.T. Atwood, W.R. Schowalter, Measurements of slip at the wall during flow of high-density polyethylene through a rectangular conduit, Rheol. Acta. 28 (1989) 134-146.

[68] F.J. Lim, W.R. Schowalter, Wall slip of narrow molecular weight distribution polybutadienes, J. Rheol. 33 (1989) 13591382.

[69] P. Mourniac, J.F. Agassant, B. Vergnes, Determination of the wall slip velocity in the flow of a SBR compound, Rheol. Acta 31 (1992) 565-574.

[70] S. Pudjijanto, M.M. Denn, A stable 'island' in the slip-stick region of linear low-density polyethylene, J. Rheol. 38 (1994) 1735-1744.

[71] J.M. Piau, N. El Kissi, Measurement and modeling of friction in polymer melts during macroscopic slip at the wall, J. Non-Newtonian Fluid Mech. 54 (1994) 121-142.

[72] J.D. Henson, M.E. Mackay, Effect of gap on the viscosity of monodisperse polystyrene melts: slip effects, J. Rheol. 34 (1995) 359-373.

[73] T.J. Person, M.M. Denn, The effect of die materials and pressure-dependent slip on the extrusion of linear low density polyethylene, J. Rheol. 41 (1997) 249-265.

[74] S.G. Hatzikiriakos, I.B. Kazatchkov, D. Vlassopoulos, Interfacial phenomena in the capillary extrusion of metallocene polyethylenes, J. Rheol. 41 (1997) 1299-1316.

[75] J. Perez-Gonzalez, L. Perez-Trejo, L. De Vargas, O. Manero, Inlet instabilities in the capillary flow of polyethylene melts, Rheol. Acta 36 (1997) 677-685.

[76] C. Venet, B. Vergnes, Experimental characterization of sharkskin in polyethylenes, J. Rheol. 41 (1997) 873-892.

[77] X. Yang, H. Ishida, S-Q. Wang, Wall slip and absence of interfacial flow instabilities in capillary flow of various polymer melts, J. Rheol. 42 (1998) 63-80.

[78] V. Mhetar, L.A. Archer, Slip in entangled polymer solutions, Macromolecules 31 (1998) 6639-6649.

[79] J.K. Hunter, M. Slemrod, Viscoelastic fluid flow exhibiting hysteritic phase changes, Phys. Fluids. 26 (1983) 2345-2351.

[80] E.B. Bagley, I.M. Cabott, D.C. West, Discontinuity in flow curve of polyethylene, J. App. Phys. 29 (1958) 109-110.

[81] R.W. Myerholtz, Oscillating flow behavior of high-density polyethylene melts, J. App. Polym. Sci. 11 (1967) 687-698.

[82] H.A. Barnes, A review of the slip (wall depletion) of the polymer solutions, emulsions and particle suspensions in viscometers: its cause, character and cure, J. Non-Newtonian Fluid Mech. 56 (1995) 221-251. 